\title{
On the Formation and Propagation of Hydrothermal waves in Liquid Layers with Phase Change
}

\author{
Marcello Lappa ${ }^{1}$ \\ ${ }^{1}$ Department of Mechanical and Aerospace Engineering, University of Strathclyde, James Weir \\ Building, 75 Montrose Street, Glasgow, G1 1XJ, UK - email: marcello.lappa@strath.ac.uk
}

Abstract: This paper reports on a numerical model expressly developed to inquire about the role of solidification in determining the properties of the emerging surface-tension-driven flow in typical models of oxide crystal growth. Following earlier efforts in the literature, we consider substances which have already enjoyed a widespread consideration for such a kind of studies, i.e. sodium nitrate $\left(\mathrm{NaNO}_{3}, \mathrm{Pr}=8\right)$ and succinonitrile $(\mathrm{SCN}, \mathrm{Pr}=23)$. Specific numerical examples are expressly elaborated and presented to provide inputs for an increased understanding of the main cause-andeffect relationships driving fluid flow and determining its properties. It is shown that, by interfering with the hydrothermal mechanism, namely the preferred mode of instability of Marangoni flow over a wide range of substances and conditions, solidification contributes to the chaoticity of the system by increasing the complexity of the emerging patterns and enriching the spectral content of the flow.

\section{Introduction}

Over recent years, the progressive refinement of manufacturing techniques in the main field of crystal growth has enabled the production of transparent materials with "controlled" microscopic structures suitable for a variety of advanced applications. Despite such improvements, however, relevant industrial production methods are still adversely affected by undesired fluid-dynamic instabilities that develop when the material is in a liquid state (Shi and Imaishi [1]; DelgadoBuscalioni [2,3]; Shevtsova et al. [4,5]; Schwabe and Benz [6]; Schwabe et al. [7]; Kaddeche et al. [8] and references therein). Indeed, the "purity" and "perfection" of the resulting crystalline structures are in general crucial factors for the exploitation of such materials and the success of related applications. In turn, these factors (both) are strongly dependent on the (fluid-dynamic) phenomena that occur when the transition from an initial liquid state (melt) to the final solid phase takes place.

There is general consensus that the temperature field in the melt can give rise to gradients of density, or surface tension, or both, and that fluid motion resulting from such gradients can substantially interfere with the growth process. These thermally-induced fluid flows can indeed interact with dynamic boundary conditions such as an advancing solidification front. The resultant fluid motion is often complex, but always critical to crystal quality (Pimputkar and Ostrach [9]; Müller [10]).

Examples of oxide materials of current interest are $\mathrm{MgAl}_{2} \mathrm{O}_{4}$ (spinel), $\mathrm{Al}_{2} \mathrm{O}_{3}$ (sapphire), $\mathrm{Y}_{3} \mathrm{Al}_{5} \mathrm{O}_{12}$ (yttrium aluminium), $\mathrm{YAlO}_{3}$ (yttrium orthoaluminate), $\mathrm{Gd}_{3} \mathrm{Ga}_{5} \mathrm{O}_{12}$ (gadolinium gallium garnet), $\mathrm{LiTaO}_{3}$ (lithium tantalate). The Prandtl number of these materials when they are in the liquid state is larger than one $(\operatorname{Pr}>1)$, which can be regarded as a clear distinguishing mark with respect to standard semiconductors or liquid metals. Related applications include solid state lasers, magnetic 
bubble device substrates, insulating substrates for semiconductors, and monolithic crystal filters. Another category of similar substances is represented by the so-called transparent conductive oxides (TCOs). These oxides are widely used as transparent "metallic" electrodes or solar cells and flat panels including liquid crystal displays (LCDs) and organic light emitting diodes (OLEDs). Because of their unique properties of coexistence of "optical transparency in visible region" and "controllability of electronic conduction from insulator to metal" (Ohta et al. [11]), such materials have enjoyed a widespread use in a variety of power saving opto-electrical circuitries and technological applications (Stadler [12]).

Control of convective instabilities occurring when the considered material is in a liquid state, in general, is regarded as an essential topic from a "product quality" perspective. Although a plethora of studies have been appearing over recent years motivated, completely or in part, by the need to elaborate new means to mitigate such instabilities (and hence to produce single crystals of higher quality), unfortunately, most of these studies were focusing on idealized geometries with limited translational applicability to effective production methods. A large cross section of fundamental research on these topics has been reviewed, e.g., by $[13,14]$.

For the case of thermogravitational (buoyancy) convection, a first step in this direction was undertaken by Coriell et al. [15], who considered the stability of the vertical parallel flow between a crystal-melt interface and a wall held at a temperature above the melting point of the crystal for Prandtl numbers ranging from $10^{-2}$ to $10^{2}$. Three modes of instability were identified accordingly: (a) a buoyant mode, (b) a shear mode, and (c) a coupled crystal-melt mode. The buoyant and shear modes were found to be similar to those that occur for flow between two vertical rigid walls held at different temperatures. For Prandtl numbers greater than approximately two, the coupled crystalmelt mode was observed at a lower Grashof number than the other two modes. Detailed results were presented for succinonitrile $(\operatorname{Pr}=22.8)$ and lead $(\operatorname{Pr}=0.0225)$.

For the other limiting situation in which the parallel flow is horizontal instead of vertical, Lan et al. [16] conducted a stability and bifurcation analysis with relevance to the classical (open-boat) Bridgman method. Their model accounted for two-dimensional heat transfer and fluid flow in a molten semiconductor material $(\operatorname{Pr}<<1)$, related morphological evolution of the growth interface, and heat diffusion in the crystal. The stability of various families of (flow) solutions was examined in the framework of a linear stability approach. The bifurcation diagrams were found to be slightly changed due to interface deformation (with respect to the idealized case with fixed geometry). Nevertheless, no changes were detected in the qualitative characteristics of the instability, i.e. its physical nature.

This research framework was further expanded by Bertrand et al. [17], Tönhardt and Amberg [18], El Ganaoui [19], Semma et al. [20], Stickland et al. [21] and Wang et al. [22] on the basis of different numerical methods (each with its own advantages and bottlenecks) able to address the solution of the Navier-Stokes and energy equation in their complete (non-linear and multiphase) formulation. 
Some studies have been also appearing where the interaction between the solidification process and the other dominant kind of convection in typical crystal production processes, namely the so-called Marangoni flow (or thermocapillary convection), was considered. In such a context, as an example, Shyy and Chen [23]) and Lan and Kou [24] were among the first investigators to carry out combined simulations for the so-called floating zone technique. The latter authors compared the typical dynamics emerging for high Prandtl-number materials, e.g., $\mathrm{NaNO}_{3}$, and low Prandtlnumber materials, such as silicon ( $\mathrm{Si}$ ). The unknown shapes of the melt/gas, melt-crystal and meltfeed interfaces were calculated for different situations: (a) conduction only, (b) natural convection and (c) hybrid Marangoni and buoyancy flow. Marangoni convection was found to increase the convexity of the melt-crystal interface and reduce the maximum surface temperature, this effect being more pronounced in the high-Pr melt case $\left(\mathrm{NaNO}_{3}\right)$ than in in the low-Pr melt case $(\mathrm{Si})$ (though it should be mentioned Marangoni convection was observed to be far stronger in the $\mathrm{Si}$ melt).

Using succinonitrile (SCN) as work fluid (a paradigmatic substances for the study of such dynamics owing to its relatively low melting temperature, reduced reactivity and transparency to visible light), Cerimele et al. [25] concentrated on the horizontal Bridgman solidification process where the Marangoni effect emerges at the open top of a parallelepipedic crucible. Additional existing fundamental research on this topic has been reviewed by Amberg and Shiomi [26] and Tsukada et al. [27]. Along these lines, it is also worth citing the very interesting experimental study by Matsunaga and Kawamura [28], who, yet for the case of succinonitrile, focused expressly on the influence of convection on crystal shape. Similarly, Hong et al. [29] visualized Marangoni

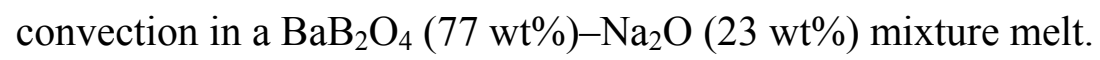

Unfortunately, analyses of such a kind are rather sparse and rare as most of past studies examined either crystal growth or thermocapillary (Marangoni) convection as disjoint subjects (typically on the basis of one-way coupling approaches, i.e. crystals with fixed shape and fixed boundary conditions used to study the response of the fluid system to the application of well-defined stimuli in terms of imposed temperature or concentration gradients).

For the specific case of two-way coupling methods (in which fluid-dynamic and phase-change aspects are fully coupled), it is worth citing Lan and Chian [30] and Lappa and Savino [31] who performed some numerical studies for circumstances in which the Marangoni flow is in supercritical conditions. However, these authors limited to the case of semiconductor melts $(\operatorname{Pr}<<1)$ in floating zones. It is a well-known fact (see, e.g., Lappa [32]) that for these values of the Prandtl number, the typical instabilities of Marangoni flow display notable differences with respect to those expected for high-Pr materials such as oxide melts or succinonitrile.

In the case of liquid layers or shallow rectangular cavities, as an example, the typical supercritical convective modes of two-dimensional parallel Marangoni flow (the so-called return flow solution, originally considered by Smith and Davis [33]) are represented by hydrothermal waves (HTW). It is known that these waves have a different direction of propagation according to the Prandtl number. They are nearly perpendicular to the basic state for low-Pr materials, i.e. the disturbance propagates 
almost exactly in the spanwise direction (the wave has a longitudinal wavefront in such a case); and nearly parallel to the surface flow for high-Pr materials, i.e. the disturbance propagates almost exactly in the upstream direction with respect to the surface flow (i.e. from the cold side to the hot side, the waveform in such a case being transverse). In the latter case, two-dimensional (2D) simulations can be used to simulate the real phenomenon with an acceptable approximation. There are several numerical solutions along these lines, see, e.g., Peltier and Biringen [34], Xu and Zebib [35], Shevtsova et al. [36], Tang and Wu [37], Lappa [38]. Simulations expressly dedicated to the case of succinonitrile or other convenient fluids (such as $\mathrm{NaNO}_{3}$ or silicone oils used to mimic the typical properties of oxide materials) are also available (Bucchignani and Mansutti [39]). Important extensions to the original work by Smith and Davis [33] and the later weakly nonlinear analysis by Smith [40] were provided by Priede and Gerbeth [41], who analysed the effect of various thermal boundary conditions on the linear stability of the parallel-flow solution. In particular, they concentrated on the more realistic case in which the adiabatic bottom is replaced by a conducting boundary (to mimic an external metallic container) with the free liquid-gas surface retaining an adiabatic behaviour. Some other interesting results were presented recently by Lappa [42], who focused on the emergence of hydrothermal waves in shallow cavities with an obstruction located on the bottom (a forward-facing step, a backward-facing step or a combination of both geometrical features).

None of these studies, however, examined the interplay of these modes of convection with solidification. Given the absence of numerical studies addressing such problems for high-Pr liquids (the molten form of transparent oxide materials), in the present work we concentrate expressly on the case of parallel Marangoni flow and related supercritical states represented by HTWs.

Following earlier efforts in the literature, we consider the typical substances which have already been widely used as "work" fluids for such a line of inquiry, that is sodium nitrate $\left(\mathrm{NaNO}_{3}, \operatorname{Pr}=8\right)$ and succinonitrile $(\mathrm{SCN}, \mathrm{Pr}=23)$. In particular, we provide (original) numerical examples specifically conceived to cover heretofore unseen dynamics. Indeed, the present work has been carried out under the idea or belief that advanced simulations may provide insights into still poorly known mechanisms and help expedite companion experimental studies (leaving aside for a while the experimental counterparts or the effective industrial applications, it is clear that conducting numerical simulations can become worth in its own rights because they can be used to move through unexplored territories).

\section{Mathematical Model and Numerical Method}

\subsection{The Geometry and related boundary conditions}

As discussed to a certain extent in the introduction, the present work aims to extend earlier studies about the properties of HTWs in liquid (plane) layers with fixed (imposed) temperature difference and fixed geometry $([34-35,37-38,42])$ to the case in which the liquid undergoes a solidification 
process (which implies a variable shape of the liquid domain and an applied temperature difference no longer constant in time). Accordingly, we consider a shallow cavity laterally delimited by two solid walls, one maintained at fixed temperature and the other with a temperatures changing in time as shown in Fig.1.

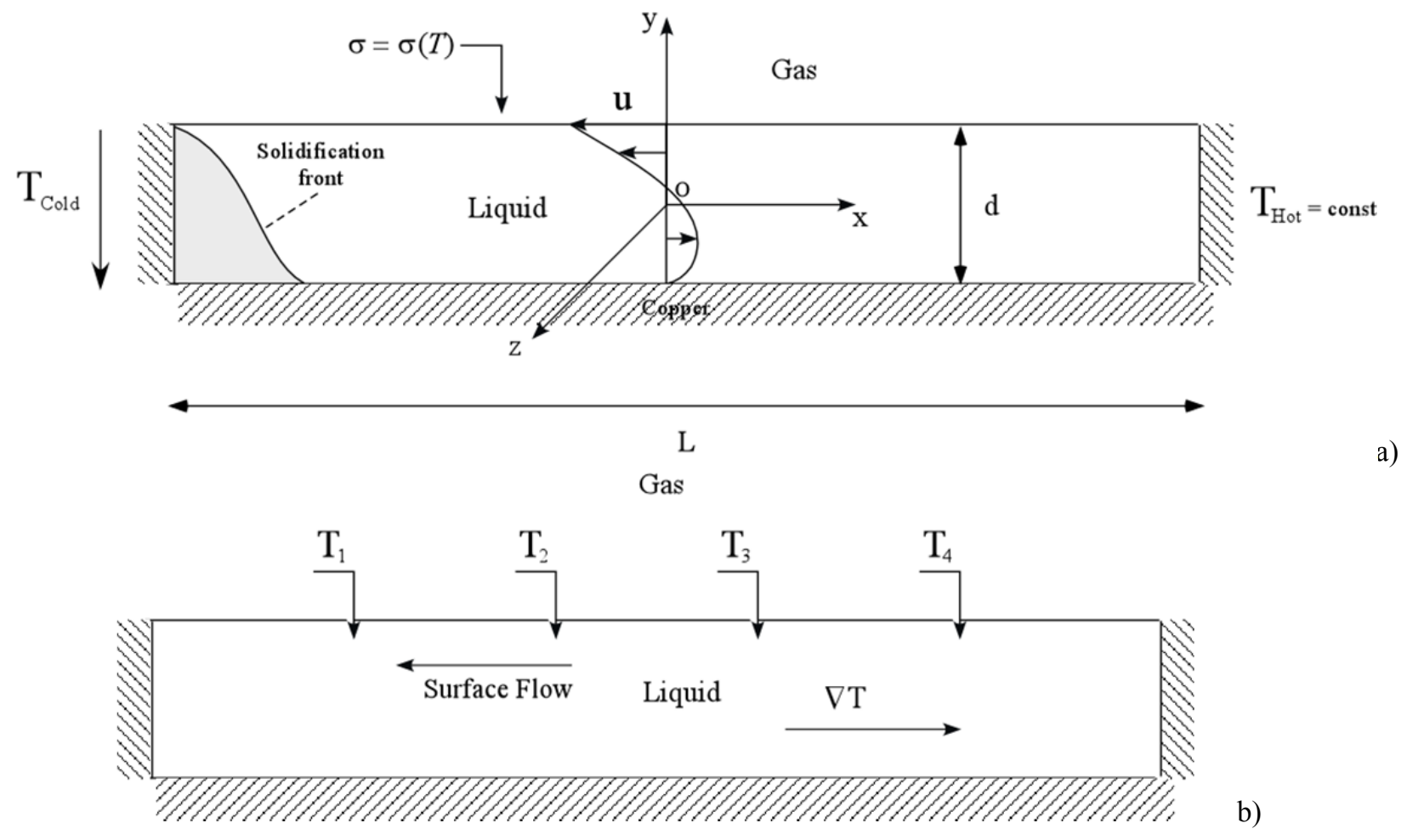

Figure 1: a) Sketch of the considered problem, b) location of thermocouples used to monitor the system response as a function of time.

The top liquid-gas interface is considered adiabatic, while the bottom wall is assumed to be made of copper (to mimic the behaviour of an ideal perfectly conducting boundary). The temperature of the right sidewall $\mathrm{T}_{\text {Hot }}$ is higher than the temperature $\mathrm{T}_{\text {Cold }}$ of the left cold boundary. Solidification takes place as the temperature $T_{\text {Cold }}$ is decreased to a value which is less than the melting temperature $T_{m}$. Though the extension of the liquid domain is not constant in time, these conditions make this system an ideal target to study the dynamics of hydrothermal waves in the presence of solidification while retaining well-controllable conditions. The streamwise domain aspect ratio $\left(A_{x}\right)$, defined as its initial length-to-depth ratio (L/d) is fixed to 20 as in earlier related works $[38,42]$. For completeness, the spanwise aspect ratio is introduced as $A_{z}=W / d$ where $W$ is the system width, i.e. its extension along the third direction $\mathrm{z}$ (assumed to be infinite when the problem is addressed in the framework of two-dimensional simulations). 


\subsection{Phase-change modelling theory: The enthalpy method}

Studying the dynamics of fluid flow interacting with a solid-liquid phase change process requires, in general, numerical methods specifically conceived to track the evolution of the phase boundary, able to account at the same time for the heat transfer within and between the two separate phases.

Enthalpy methods and similar techniques address these aspects by expressly modelling the release or absorption of latent heat. This class of methods was originally introduced by Voller and Prakash [43], Bennon and Incropera [44,45], Brent et al. [46]. Additional variants have been elaborated over the years for different systems and circumstances (see, e.g., Beckermann et al. [47]; Knoll et al. [48]; and many others). The available approaches developed to date by the different researchers are articulated essentially into two main categories: "adaptive methods" (Rao and Shyy [49]; Lan and Liang [50]; Tönhardt and Amberg [18]; Lan and Liu [51]) and "fixed-mesh techniques".

With the first approach the liquid and the solid are treated as two distinct grid domains, this being accomplished via the adoption of a finite-element strategy. In the second case, no explicit distinction is introduced between the solid and the liquid (at least from a computational point of view). Indeed, the governing equations are typically solved on a single regular grid where the different (convective and diffusive) equations terms are discretized using standard finite volume or difference stencils. This is made possible by the specific nature of the enthalpy method by which the phase change is incorporated implicitly in the algorithm by assuming that the liquid fraction is a function of temperature.

With the adaptive methods, the solution strategy has obviously to deal with a numerically generated orthogonal curvilinear coordinate system, which needs to be adjusted dynamically as the solidification front evolves in time. By contrast, with a fixed-mesh technique based on the so-called enthalpy formulation, the need to explicitly satisfy conditions at the phase front is removed and, as outlined above, one is allowed to utilize standard solution procedures which do not require mathematical manipulations and transformations (see, e.g., Udaykumur et al. [52]; Kim et al. [53]; El Ganaoui et al. [19]; Sekerka [54]; Faiez and Rezaei [55]). In the light of these examples the present study relies on the second type of approach and the related fundamental equations are introduced and discussed in the framework of the problem defined in Sect. 2.1.

With the enthalpy methods, the latent heat in the energy equation is dealt with by assigning a nodal latent heat value to each computational cell according to the "local" temperature. When phase change occurs, such a value is increased or decreased according to whether latent-heat absorption or release is taking place. From a mathematical point of view this is equivalent to the introduction of a source or a sink in the energy equation. In practice, this is achieved by defining the enthalpy as :

$\mathrm{h}=\mathrm{C}_{\mathrm{p}} \mathrm{T}+\Delta \mathrm{H}$

where $\Delta \mathrm{H}=\phi \mathrm{L}_{\mathrm{m}}, \mathrm{L}_{\mathrm{m}}$ is the latent heat, $\mathrm{C}_{\mathrm{p}}$ the specific heat coefficient and $\phi$ the liquid fraction defined as

$\phi=0$ for $\mathrm{T}<\mathrm{T}_{\text {sol }}$ 
$\phi=\frac{T-T_{\text {sol }}}{T_{\text {liq }}-T_{\text {sol }}}$ for $\mathrm{T}_{\text {sol }} \leq \mathrm{T} \leq \mathrm{T}_{\text {liq }}$

$\phi=1$ for $\mathrm{T}>\mathrm{T}_{\text {liq }}$

where $\mathrm{T}_{\mathrm{sol}}=\mathrm{T}_{\mathrm{m}}-\Delta \mathrm{T}_{\mathrm{m}}, \mathrm{T}_{\text {liq }}=\mathrm{T}_{\mathrm{m}}+\Delta \mathrm{T}_{\mathrm{m}}$ and $2 \Delta \mathrm{T}_{\mathrm{m}}$ is the temperature range representing the interval over which phase change occurs $\left(\mathrm{T}_{\text {liq }}\right.$ is the liquidus temperature at which solid formation is assumed to initiate, while $\mathrm{T}_{\mathrm{sol}}$ is the temperature at which full solidification is attained, see [43]). This is equivalent to consider an extended temperature range for solidification, which, from a physical point of view has its root in the realization $([43,44])$ that in many cases solid formation occurs as a permeable crystalline-like matrix coexisting with the liquid phase (mushy region). From a numerical point of view, a linear approximation (eq. (2b)) is typically considered for the liquid fraction since an abrupt change in the liquid fraction could delay the convergence of the numerical algorithm seriously.

Accordingly, the energy equation can be cast in compact form as:

$\frac{\partial \rho h}{\partial}=-\underline{\nabla} \cdot[\rho \underline{V h}]+\underline{\nabla} \cdot[\lambda \underline{\nabla T}]-\frac{\partial \rho\left(\phi L_{m}\right)}{\partial}$

where $\rho$ is the density, $\lambda$ is the thermal conductivity, $\underline{\mathrm{V}}$ is the flow velocity; the unsteady latent heat content term on the left side of eq. (3) is equal to zero for $\mathrm{T}<\mathrm{T}_{\text {sol }}$ and $\mathrm{T}>\mathrm{T}_{\text {liq }}$ (it influences the energy equation only within the mushy region). The reader may immediately realize the physical significance of this equation by noting that the first, second and third terms on the right hand side are the convective, diffusive and latent heat release/absorption contributions, respectively.

\subsection{The Governing equations}

We assume constant physical properties for the material with the melt behaving as an incompressible liquid having the same properties of the solid. Equation (3), together with the continuity and Navier-Stokes equations, can be written accordingly in nondimensional form scaling the lengths by a reference distance (the layer depth in our case, see Fig.1) and the velocity by the energy diffusion velocity $V_{\alpha}=\alpha / d\left(\alpha=\lambda / \rho C_{p}\right.$ is the thermal diffusivity); the corresponding scales for time and pressure can be defined, respectively, as $t_{r e f}=d^{2} / \alpha$ and $\pi_{r e f}=\rho \alpha^{2} / d^{2}$. The nondimensional conservative form of the governing balance equations for mass, momentum and energy can be finally cast in condensed form as:

$\underline{\nabla} \cdot \underline{\mathrm{V}}=0$

$\frac{\partial \underline{V}}{\partial t}=-\underline{\nabla} \pi-\underline{\nabla} \cdot[\underline{V} \underline{V}]+\operatorname{Pr} \nabla^{2} \underline{V}-\operatorname{Pr} \frac{1}{\eta_{p}} \underline{V}$ 


$$
\frac{\partial T}{\partial}+\frac{L_{m}}{C_{p}} \frac{\partial \phi}{\partial}=-\underline{\nabla} \cdot[\underline{V} T]+\nabla^{2} T
$$

where $\mathrm{V}$ and $\pi$ are the nondimensional velocity and pressure (pressure is denoted by $\pi$ ), $\operatorname{Pr}$ is the Prandtl number, defined by $\operatorname{Pr}=v / \alpha$ ( $v$ is the kinematic viscosity) whereas the temperature $T$ is kept in its dimensional form.

Despite the several advantages illustrated before, the implementation of such methods, however, is not as straightforward as one would expect. The accommodation of the zero-velocity condition, which is required as a liquid region is transformed into solid, indeed, is often regarded as a source of problems unless specific countermeasures are taken to deal properly with it. Various methods have been elaborated for such a purpose over the years (see [46]). A feature common to all such variants is the idea to "damp" velocities in computational cells that are freezing. Velocities may abruptly be set to zero. Another possible approach is based on the viscosity. The viscosity of a cell can be increased to a very large value proportional to the decrease in the latent heat content of the cell. This artifice can be used to properly couple the physical state of the material in the cell and the fluid momentum balance equation. A third possible variant (enthalpy-porosity approach) is based on the idea that computational cells that are undergoing a phase change could behave as a pseudo porous media, with the porosity $\eta_{p}$ being linked to the liquid fraction $\phi$ ranging between 1 (fully liquid cell) and 0 (fully solid cell). This strategy is used here. The term $-\operatorname{Pr} \underline{V} / \eta_{p}$ in eq. (4b), indeed, is the Darcy term (see $[45,46]$ ) expressly inserted in the momentum equation to mitigate convection in the liquid phase undergoing solidification. The permeability is generally assumed to vary according to the Carman-Kozeny equation:

$\eta_{p}=\frac{\phi^{3}}{(1-\phi)^{2}}$

In the pure solid $(\phi=0)$ and pure liquid $(\phi=1)$, eq. ( $4 \mathrm{~b})$ reduces to the appropriate limits, namely $\eta_{\mathrm{p}}=0$ and $\eta_{\mathrm{p}}=\infty$, respectively. In practice, the effect of $\eta_{\mathrm{p}}$ can be sketched as follows: in full liquid elements $1 / \eta_{\mathrm{p}}$ is exactly zero and plays no role; in elements undergoing phase change, the value of $1 / \eta_{\mathrm{p}}$ overcomes all the transient, convective and diffusive components of the momentum equation, thereby forcing it to mimic the Carman-Kozeny law. Since each of the continuum equations can be applied and solved regardless of the state of matter (solid or liquid), as anticipated, there is no need to give explicit consideration to boundaries between solid, multiphase and liquid regions.

Despite such advantages, anyhow (for the sake of completeness), it should be recalled that some of the assumptions invoked above (first of all that of constant thermodynamic properties) should be regarded as idealizations. As an example, it is known that assuming a different thermal conductivity for the solid with respect to that of the liquid can lead to an appreciable change in the time required for crystal growth. It can also have an impact on the morphology (concave or convex shape of the crystal front). Some recent studies have also appeared showing that in some cases the thermal conductivity of the solid may even behave as a non-isotropic quantity, taking different values 
according to the considered spatial direction [56]. This point, in turn, is connected to another wellknown issue relating to the interplay between the cooling rate applied to produced solidification and the porosity of the "pre-solid" phase (in the mushy region) and the solid material itself. Indeed, some consensus exists in the literature that slower cooling can produce a significantly greater tendency to formation of pores with respect to faster cooling, whereas smaller size and lower volume fraction of porosity are typically observed at higher cooling rate [57-59]. These effects can cause a non-isotropic distribution of the thermal conductivity (this quantity becoming higher where $\eta_{\mathrm{p}}$ is smaller and vice versa). For the sake of simplicity (towards the end to constraint an otherwise too large space of parameters and interdependences), these complex aspects are not taken into account by the present model.

\subsection{Surface-tension-driven effects}

In addition to the theoretical effort provided to properly account for the release or absorption of latent heat in the energy equations, a similar consideration must be given to the proper modelling of the sources of momentum, i.e. the forces driving flow in the considered conditions. In our case such forces are essentially surface-tension-related. Indeed, due to the considered temperature difference surface-tension forces $\underline{F}_{\sigma}^{T}=\underline{\nabla}_{S} \sigma\left(\underline{\nabla}_{S}\right.$ derivative tangential to the interface, $\sigma$ surface tension $)$ arise, which must be balanced by viscous stresses in the liquid.

From a mathematical point of view (assuming the dynamic viscosity of the gas surrounding the free liquid surface to be negligible with respect to the viscosity of the considered liquid) this balance condition can be written as:

$$
\underset{\underline{\tau}}{=} \cdot \underline{\hat{j}}=-\sigma_{T}(\underline{I}-\underline{j} \hat{j} \underline{j}) \cdot \underline{\nabla T}
$$

where $\sigma_{\mathrm{T}}$ is the derivative of surface tension with respect to temperature, $\underline{\tau}_{d}=2 \mu(\underline{\nabla V})_{o}^{s}$ is the dissipative part of the stress tensor, $\hat{j}$ is the unit vector perpendicular to the liquid/gas interface (directed from liquid to gas), $I_{=}$is the unity matrix and $\mu=v \rho$ is the liquid dynamic viscosity. For a planar surface, the balance above in nondimensional form simply yields:

$$
\frac{\partial \underline{V}_{S}}{\partial n}=-M a^{1} \underline{\nabla}_{S} T
$$

where $\underline{\mathrm{V}}_{\mathrm{s}}$ is the surface velocity vector and $\mathrm{Ma}^{1}=\sigma_{\mathrm{T}} \mathrm{d} / \mu \alpha$.

The additional non-dimensional parameter:

$\mathrm{Ma}=\mathrm{Ma}^{1} \Delta \mathrm{T}$ 
( $\Delta \mathrm{T}$ being the effective temperature difference at the liquid-gas interface) is the so-called Marangoni number. This condition induces fluid flow in the bulk of the liquid enforced by the tangential variation of the surface tension at the interface. A well-known feature of this kind of flow (hereafter referred to as thermocapillary or Marangoni convection) is the absence of a threshold value to be exceeded, i.e. its ability to develop even in the limit as the temperature gradient tends to zero. As soon as a temperature gradient (no matter how small) is established at the liquid-gas interface, the surface fluid is forced to move from the region where the surface tension is smaller (relatively hot) towards that with a high surface tension (relatively cold). Due to the viscosity of the fluid, this motion is transferred to the underlying fluid, i.e. the viscous coupling to the motion at the interface is the main mechanism causing initial surface flow to penetrate into the bulk.

Buoyancy, another potential source of fluid motion, is assumed to be absent or negligible in the present study. This is equivalent to stating that microgravity conditions are attained or that the socalled dynamic Bond number, defined as the ratio of the Rayleigh and Marangoni numbers, satisfies the following condition:

$$
\frac{R a}{M a}=\frac{g \rho \beta_{T}}{\sigma_{T}} d^{2}<<1
$$

where $g$ is the gravity acceleration and $\beta_{\mathrm{T}}$ is the so-called thermal expansion coefficient (as the dynamic Bond number grows quadratically with the depth of the layer, typically, buoyancy effects can be neglected in normal gravity conditions when such a depth is small)

\subsection{The Initial and Boundary conditions}

The initial conditions are:

$\mathrm{t}=0: \quad \underline{\mathrm{V}}(\mathrm{x}, \mathrm{y})=0, \mathrm{~T}(\mathrm{x}, \mathrm{y})=\mathrm{T}_{\text {liq }}+\Delta \mathrm{T} \mathrm{x} / \mathrm{A}_{\mathrm{x}}$

i.e. the liquid is in quiescent conditions with a linear temperature profile along the $\mathrm{x}$ coordinate (the temperature is $\mathrm{T}_{\text {Cold }}=\mathrm{T}_{\text {liq }}$ on the cold sidewall and $\mathrm{T}_{\mathrm{Hot}}=\mathrm{T}_{\text {liq }}+\Delta \mathrm{T}$ on the other side).

When, for $\mathrm{t}>\mathrm{t}^{*}$ the solidification process starts $\left(\mathrm{t}^{*}\right.$ is the instant at which the cooling ramp is applied to the cold sidewall and solidification begins), the thermal boundary conditions read:

$\mathrm{T}_{\text {Cold }}=\mathrm{T}_{\text {liq }}-\mathrm{c}\left(\mathrm{t}-\mathrm{t}^{*}\right)$

where $\mathrm{c}$ is a constant defined as $\mathrm{c}=\mathrm{kt}_{\mathrm{ref}}, \mathrm{k}$ being the dimensional ramping rate $\left({ }^{\circ} \mathrm{C} / \mathrm{s}\right)$

$\mathrm{T}_{\mathrm{Hot}}=\mathrm{T}_{\text {liq }}+\Delta \mathrm{T}$ 
The temperature profile for the bottom boundary (assumed to be made of copper) is determined via solution of the one-dimensional heat equation

$$
\frac{\partial T}{\partial t}=\tilde{\alpha} \nabla^{2} T
$$

with $\mathrm{T}=\mathrm{T}_{\text {Cold }}$ and $\mathrm{T}=\mathrm{T}_{\mathrm{Hot}}$ at $\mathrm{x}=-\mathrm{A} / 2$ and $\mathrm{x}=\mathrm{A}_{\mathrm{x}} / 2$, respectively, where $\tilde{\alpha}=\alpha / \alpha_{\mathrm{Cu}}$ (in this way we assume there dynamic thermal boundary conditions, which tend ideally those that would be maintained by a perfectly conducting wall when steady conditions are attained). Moreover:

$\partial \mathrm{T} / \partial \mathrm{y}=0$ at $\mathrm{y}=1 / 2$

Obviously, such conditions must be enriched with the adequate kinematic boundary conditions, which, in the light of the arguments elaborated in the earlier sections and by indicating with $\mathrm{u}, \mathrm{v}$ and $\mathrm{w}$ the velocity components along $\mathrm{x}, \mathrm{y}$ and $\mathrm{z}$, respectively, reduce to

$\mathrm{u}=0, \mathrm{v}=0$ and $\mathrm{w}=0$ at $\mathrm{x}= \pm \mathrm{A}_{\mathrm{x}} / 2$

$\mathrm{u}=0, \mathrm{v}=0$ and $\mathrm{w}=0$ at $\mathrm{y}=-1 / 2$

$\mathrm{v}=0$ and $\partial \mathrm{u} / \partial \mathrm{y}=-\mathrm{Ma}^{1} f(\mathrm{x}) \partial \mathrm{T} / \partial \mathrm{x}, \partial \mathrm{w} / \partial \mathrm{y}=-\mathrm{Ma}^{1} f(\mathrm{x}) \partial \mathrm{T} / \partial \mathrm{z}$ at $\mathrm{y}=1 / 2$

$\mathrm{u}=0, \mathrm{v}=0$ and $\mathrm{w}=0$ and $\partial \mathrm{T} / \partial \mathrm{z}=0$ at $\mathrm{z}= \pm \mathrm{A}_{\mathrm{z}} / 2$

the rationale behind the function $f(x)=\phi^{2}$ appearing at the right member of eq. (12c) can be found in Kasperski and Labrosse [60]. In general, this function is used to fix the singularity that affects Marangoni flow in the region where free and solid boundaries meet. This singularity, which becomes effective for high Prandtl number liquids $(\operatorname{Pr}>1$, for which strong thermal boundary layers appear close to the cooled wall with thickness scaling according to $\left.\ell=(\operatorname{Pr} \operatorname{Re})^{-1 / 3}=(\mathrm{Ma})^{-1 / 3}\right)$, can seriously jeopardize the physical consistency of the numerical solution and also prevent convergence in some circumstances. From a mathematical point of view, this issue can be fixed if the Marangoni stresses vanish at the solid walls while keeping a continuous behaviour (the expression $f(x)=\phi^{2}$ is used just to satisfy this requirement). Among other things, in the case of solidification problems, the use of the regularization condition (12c) near the melt/solid fronts has also a physical correspondence or significance; as pointed out by [60], in fact, the viscosity must tend to an infinite value on the freezing front (i.e. $\mathrm{Ma} \rightarrow 0$ locally). 


\subsection{The Numerical method}

A primitive-variable approach has been used to solve numerically the governing equations and the related boundary conditions. Following typical past implementations of such a solution strategy, proper coupling of pressure and velocity fields has been enforced in the framework of a finitedifferences technique resorting to a staggered arrangement of fluid-dynamics variables on the computational grid. The details of the solution strategy are not discussed extensively here, the interested reader being referred to various books and articles in the literature for an exhaustive treatment. The present code was repeatedly validated in previous studies (see, e.g., Lappa [38] for a validation study concerning the ability of the code to properly capture the instabilities of Marangoni flow in liquid layers and [31] for a similar assessment concerning its solidification kernel). Here we content ourselves with providing some useful information about the grid refinement analysis and the strategy that we had to implement to guarantee a proper resolution of all spatial and temporal scales involved in the considered phenomena.

\begin{tabular}{rl}
\hline \hline Grid $\mathrm{N}_{\mathrm{x}} \times \mathrm{N}_{\mathrm{y}}$ & $\Omega_{\mathrm{HTW}}$ \\
\hline \hline $200 \times 20$ & 50.4 \\
$400 \times 20$ & 48.0 \\
$300 \times 30$ & 47.5 \\
$400 \times 30$ & 47.2 \\
$600 \times 30$ & 47.1 \\
\hline \hline
\end{tabular}

Table I: Grid Refinement Study: Angular frequency of the Hydrothermal Wave as a function of mesh resolution $\left(\operatorname{Pr} \cong 15, \mathrm{~A}_{\mathrm{x}}=20, \mathrm{Ma}=3 \times 10^{4}, 2 \mathrm{D}\right.$ simulations, adiabatic bottom wall).

As representative conditions for the spatial grid refinement study, we have considered an intermediate value of the Prandtl number $(\operatorname{Pr}=15)$ and a relatively high value of the Marangoni number $\left(\mathrm{Ma}=3 \times 10^{4}\right)$ for which the solution is expected to be affected by the presence of lateral thermal boundary layers (whose proper resolution is essential to ensure grid independency). As sensitive parameters to check grid independency, we have considered the angular frequency of the hydrothermal wave. The minimum mesh required to guarantee independency of such a frequency from the used spatial resolution has been found to be 30 points per unit nondimensional length. Accordingly, for the 2D simulations a mesh with 600 points in the $\mathrm{x}$ direction and 30 points in the $\mathrm{y}$ direction has been used (i.e. 30 grid points per unit nondimensional length along both spatial horizontal and vertical directions, see Table I). The criterion for the selection of the temporal resolution (i.e. the time integration step) has been based on the combination of three independent requirements:

$$
\Delta t_{1} \leq \frac{1}{2 \operatorname{Pr}} \frac{\Delta x^{2} \Delta y^{2}}{\Delta x^{2}+\Delta y^{2}}
$$


$\Delta t_{2} \leq \frac{1}{2} \frac{\Delta x \Delta y}{u_{\max } \Delta y+v_{\max } \Delta x}$

$\Delta t_{3} \leq \frac{1}{2} \tilde{\alpha} \Delta x^{2}$

$\Delta t=\min \left\{\Delta t_{1}, \Delta t_{2}, \Delta t_{3}\right\}$

where the first two inequalities are the well-known conditions for the numerical stability of the diffusive and convective equation terms, respectively, while the third one concerns the numerical stability of the heat equation used to determine the distribution of temperature in the bottom (conducting) wall made of copper. For the limited set of 3D simulations reported in the present work as well, the first two requirements have been replaced by a single constraint to be applied for the stability of the diffusive terms, namely,

$\Delta t \leq \frac{1}{2 \operatorname{Pr}} \Delta \xi^{2}$

where $\Delta \xi=\min \{\Delta x, \Delta y, \Delta z\}$

\section{Results}

The properties of the considered substances and materials are given in Table II.

These materials are often used for solidification experiments and related lines of inquiry, because of their relatively low melting point, transparency to visible light, well-known physical properties and the solidification behaviour, which closely resembles that of many oxide materials.

TABLE IIa: $\mathrm{NaNO}_{3}(\mathrm{Pr}=8)$ Liquid-solid system properties (after Melnikov et al. [61]; Latent Heat after Nissen and Meeker [62]).

Fluid $\left(\mathrm{NaNO}_{3}\right)$ density $\rho, \mathrm{kg} \mathrm{m}^{-3}$

1904

Melting temperature $\mathrm{T}_{\text {liq }},{ }^{\circ} \mathrm{C}$

308

Solidification temperature $\mathrm{T}_{\text {sol }},{ }^{\circ} \mathrm{C}$

307

Kinematic viscosity $v, \mathrm{~m}^{2} \mathrm{~s}^{-1}$

$1.27 \times 10^{-6}$

Thermal diffusivity $\alpha, \mathrm{m}^{2} \mathrm{~s}^{-1}$

$1.58 \times 10^{-7}$

Surface tension derivative $\sigma_{\mathrm{T}}, \mathrm{Nm}^{-1} \mathrm{~K}^{-1}$

$7 \times 10^{-5}$

Latent Heat $\mathrm{L}_{\mathrm{m}}, \mathrm{J} \mathrm{Kg}^{-1}$

$1.78 \times 10^{5}$

$\mathrm{C}_{\mathrm{p}}, \mathrm{J} \mathrm{Kg}^{-1} \mathrm{~K}^{-1}$

1880 
TABLE IIb: SCN ( $\mathrm{Pr}=23$ ) Liquid-solid system properties (after Chen et al. [63]; Surface tension derivative after Matsunaga and Kawamura [28])

\begin{tabular}{ll}
\hline \hline Fluid (Succinonitrile) density $\rho, \mathrm{kg} \mathrm{m}^{-3}$ & 998 \\
Melting temperature $\mathrm{T}_{\mathrm{liq}},{ }^{\circ} \mathrm{C}$ & 58.58 \\
Solidification temperature $\mathrm{T}_{\mathrm{sol}},{ }^{\circ} \mathrm{C}$ & 57.58 \\
Kinematic viscosity $\mathrm{v}, \mathrm{m}^{2} \mathrm{~s}^{-1}$ & $2.6 \times 10^{-6}$ \\
Thermal diffusivity $\alpha, \mathrm{m}^{2} \mathrm{~s}^{-1}$ & $1.13 \times 10^{-7}$ \\
Surface tension derivative $\sigma_{\mathrm{T}}, \mathrm{Nm}^{-1} \mathrm{~K}^{-1}$ & $1.06 \times 10^{-4}$ \\
Latent Heat $\mathrm{L}_{\mathrm{m}}, \mathrm{J} \mathrm{Kg}^{-1}$ & $4.79 \times 10^{4}$ \\
$\mathrm{C}_{\mathrm{p}}, \mathrm{J} \mathrm{Kg}^{-1} \mathrm{~K}^{-1}$ & 1990 \\
\hline \hline
\end{tabular}

TABLE IIc: Copper Thermal diffusivity

Thermal diffusivity Copper $\alpha_{\mathrm{Cu}}, \mathrm{m}^{2} \mathrm{~s}^{-1}$

$1.11 \times 10^{-4}$

The depth of the layer is assumed to be $\mathrm{d}=10^{-2} \mathrm{~m}$ for all cases which gives $\mathrm{Ma}^{1}=1832 \mathrm{~K}^{-1}, \mathrm{t}_{\mathrm{ref}}=633$ $\mathrm{s}$ and $\mathrm{Ma}^{1}=3615 \mathrm{~K}^{-1}, \mathrm{t}_{\mathrm{ref}}=885 \mathrm{~s}$ for $\mathrm{NaNO}_{3}$ and $\mathrm{SCN}$, respectively. Moreover, as implicitly defined by eq. (10a), we assume as initial temperature on the cold side $\mathrm{T}_{\text {cold }}=\mathrm{T}_{\text {liq }}$ (i.e. the melting temperature shown in Table II for both substances). By using as initial temperature differences $\Delta \mathrm{T}=11^{\circ} \mathrm{C}$ and $\Delta \mathrm{T}=8.3^{\circ} \mathrm{C}$, for $\mathrm{NaNO}_{3}$ and $\mathrm{SCN}$, we obtain accordingly two "representative" values of the Marangoni number, namely $\mathrm{Ma} \cong 2 \times 10^{4}$ and $\mathrm{Ma} \cong 3 \times 10^{4}$, respectively. The corresponding temperatures on the hot wall are $\mathrm{T}_{\mathrm{Hot}}=319^{\circ} \mathrm{C}$ and $\mathrm{T}_{\mathrm{Hot}}=66.9^{\circ} \mathrm{C}$ for $\mathrm{NaNO}_{3}$ and $\mathrm{SCN}$, respectively. As explained in Sect. 2.5, such temperatures are kept fixed, the solidification process being induced by a decrease in the temperature at the cold wall.

\subsection{The Hydrothermal Wave}

For the above conditions, assuming the temperatures at the two sidewalls to be fixed (i.e. no solidification process being enabled) hydrothermal waves develop for both substances with angular frequencies $\Omega_{\mathrm{HTW}}=33.2$ and $\Omega_{\mathrm{HTW}}=54.8$ for $\mathrm{NaNO}_{3}$ and succinonitrile, respectively.

As shown by two-dimensional simulations, the presence of the hydrothermal wave is revealed by the propagation of well-defined convective structures from the cooled side towards the heated one, i.e. in the upstream direction as originally predicted by [33]. 


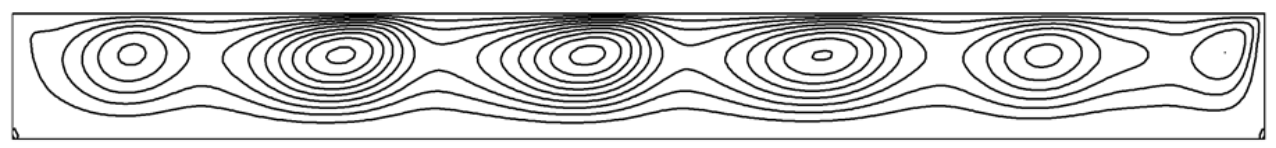

a)

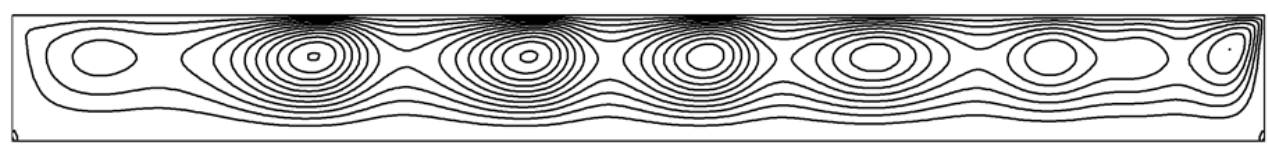

b)

Figure 2: Snapshot of Hydrothermal wave for adiabatic surface and conducting bottom wall $\left(\psi_{\max }=60\right.$, interlevel $\left.\Delta \psi \cong 4.3\right)$ : a) $\left.\operatorname{Pr}=8, \mathrm{Ma}=2 \times 10^{4}, \mathrm{~b}\right) \operatorname{Pr}=23, \mathrm{Ma}=3 \times 10^{4}$

As evident in Fig. 2, the hydrothermal wave undergoes some mitigation close to the hot wall; when a roll "disappears" in proximity to the hot side, a new roll nucleates at the cold side thereby keeping constant (on average) the overall number $\mathrm{N}$ of vortex cells travelling in the cavity at any instant ( $\mathrm{N} \cong 6$ and $\mathrm{N} \cong 7$ for $\mathrm{NaNO}_{3}$ and $\mathrm{SCN}$, respectively).

The above scenario may be regarded as a good description of the typical dynamics produced by the transverse component of the hydrothermal wave.

In general, such waves have both transverse (in the plane of the basic flow) and longitudinal components (perpendicular to the basic flow). The ability of two-dimensional simulations to retain the "essence" of the physical process (with marginal losses or departures with respect to reality) can be assessed by taking a look at Fig. 3 and Fig. 4 showing the typical outcome of equivalent (i.e. for the same set of parameters) three-dimensional (3D) simulations (note that in such figures the nondimensional temperature has been introduced as $\left.\left(\mathrm{T}-\mathrm{T}_{\text {liq }}\right) / \Delta \mathrm{T}\right)$. By inspection of Figures $3 \mathrm{c}$ and $4 \mathrm{c}$, it becomes clear that the inclination angle $\theta$ of such waves with respect to the basic flow is relatively small $\left(\theta \cong 29^{\circ}\right.$ and $\theta \cong 19^{\circ}$ for $\mathrm{NaNO}_{3}$ and $\mathrm{SCN}$, respectively, in excellent agreement with the results of the linear stability analysis by Priede and Gerbeth [41] for the case of conducting conditions on the bottom wall). Moreover, as shown in Figs. $3 b$ and $4 b$ the surface distribution of temperature can be considered essentially two-dimensional for such values of the Prandtl number (the larger the value of Pr, the less significant the departure from a purely two-dimensional field). Though it is clear that future work shall be devoted to the fully three-dimensional simulations, the above arguments provide a justification for our initial decision (motivated by the express intent to save computational time) to base the present analysis of the solidification process (and related descriptions in the remainder of this paper) entirely on two-dimensional computations. 


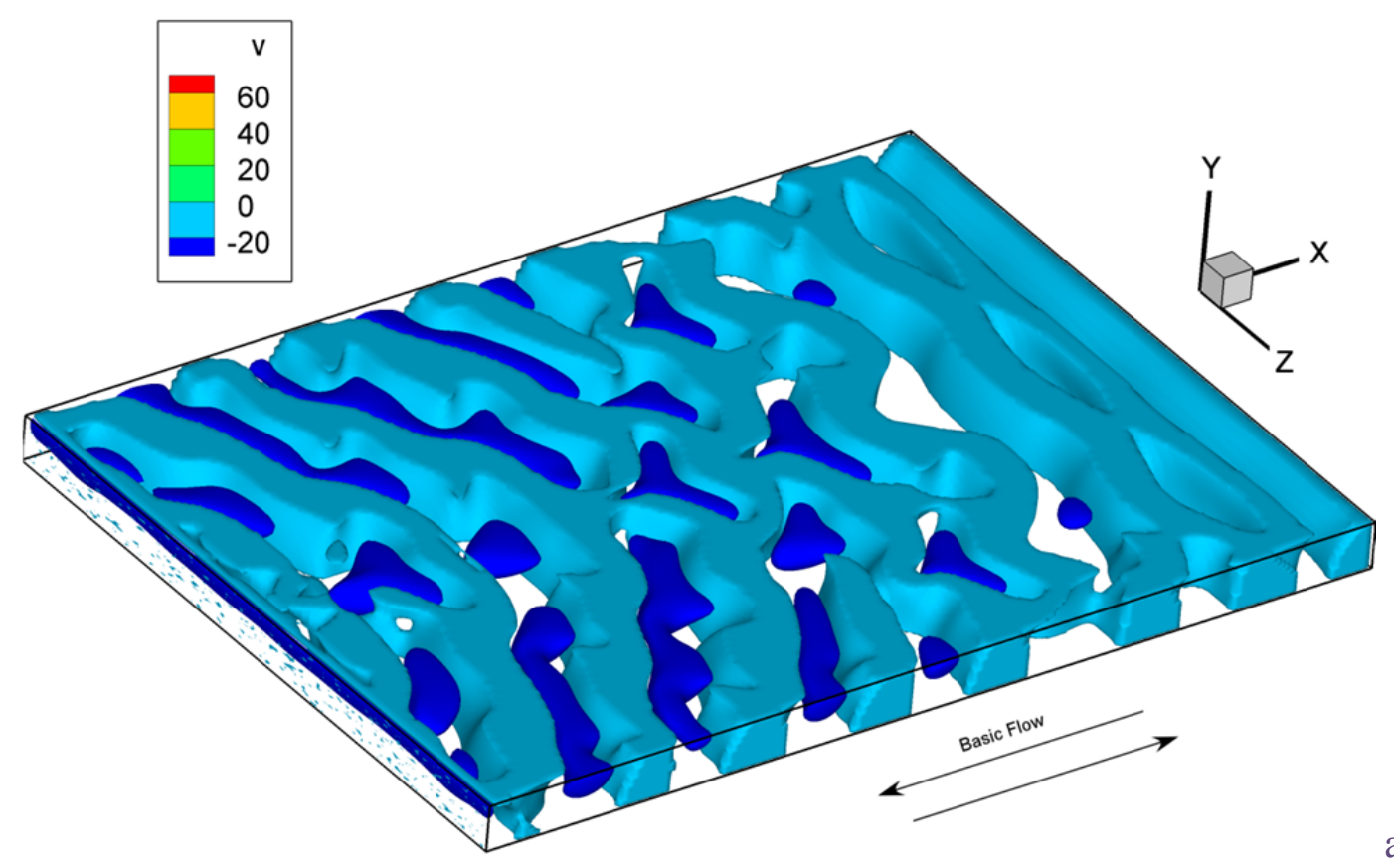

a)

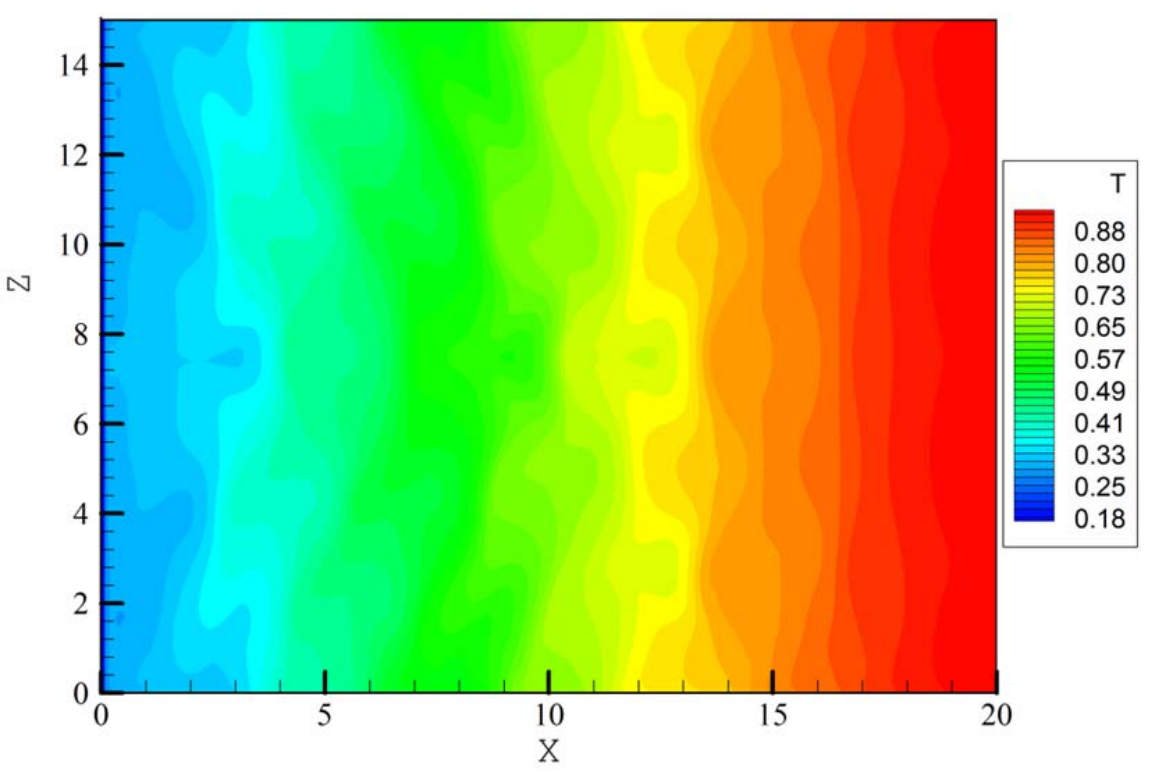

b) 


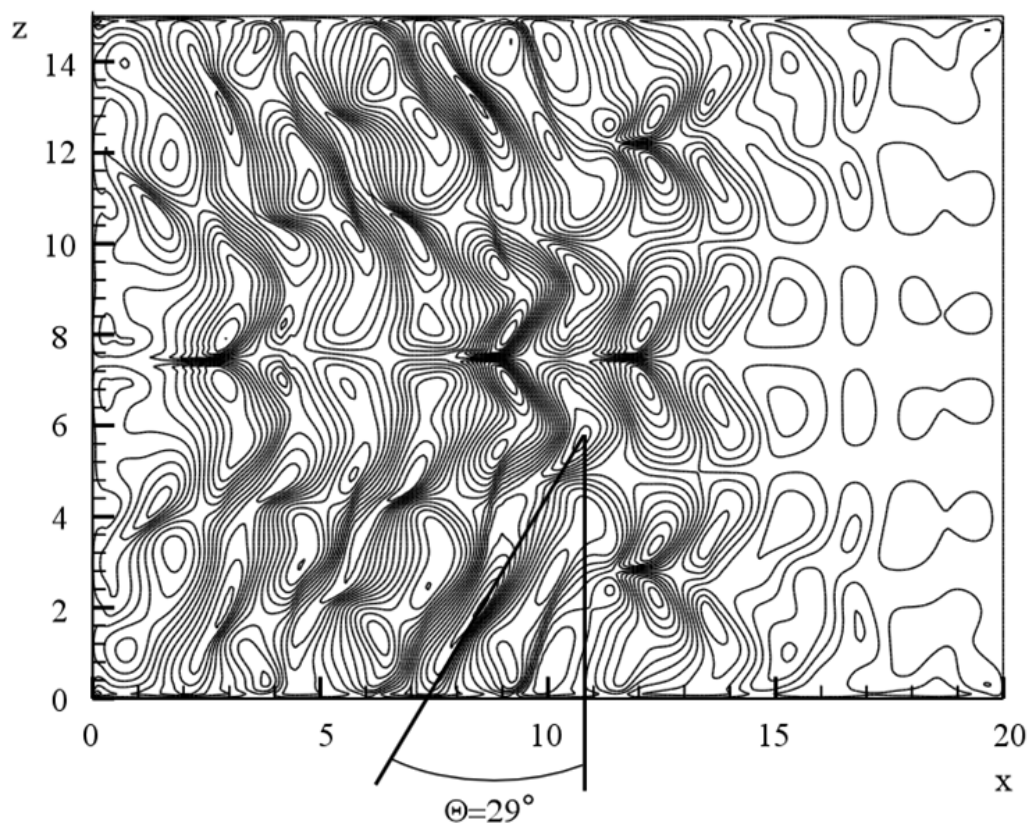

c)

Figure 3: Structure of the thermofluid-dynamic field in a 3D layer of $\mathrm{NaNO}_{3}$ with conducting conditions on the bottom wall, isothermal walls at $\mathrm{x}=0$ and $\mathrm{x}=20$, adiabatic surface and adiabatic walls at $\mathrm{z}=0$ and $\mathrm{z}=15\left(\mathrm{~A}_{\mathrm{x}}=20, \mathrm{~A}_{\mathrm{z}}=15, \mathrm{Pr}=8, \mathrm{Ma}=2 \times 10^{4}, \mathrm{t}=4\right)$ : a) isosurfaces (snapshot) of the vertical component of velocity revealing the presence of transverse rolls, which travel from the cold side towards the hot side, b) snapshot of surface temperature distribution, c) distribution of the longitudinal component of velocity $(w)$ on the free interface revealing the inclination angle of travelling waves with respect to the basic flow.

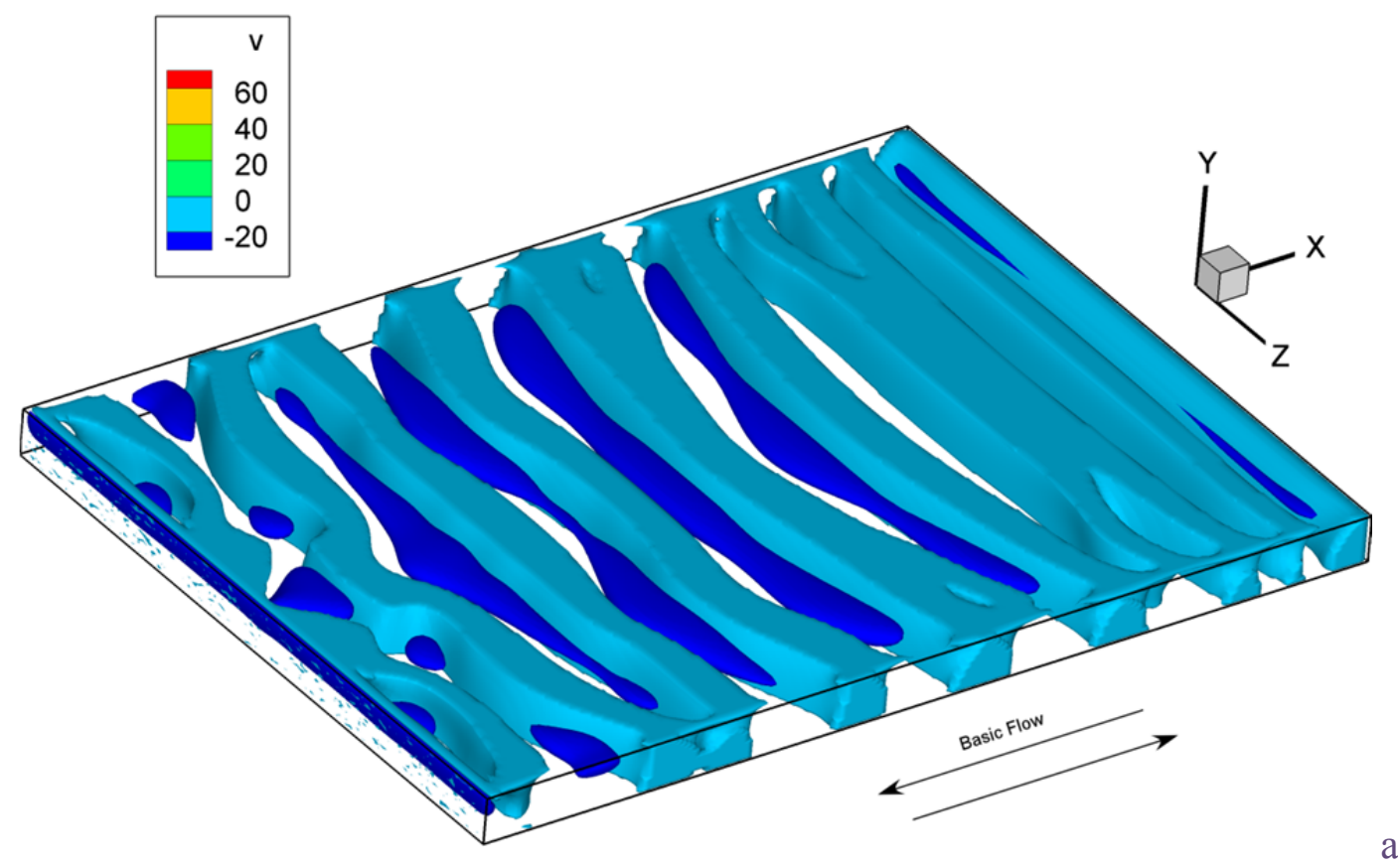




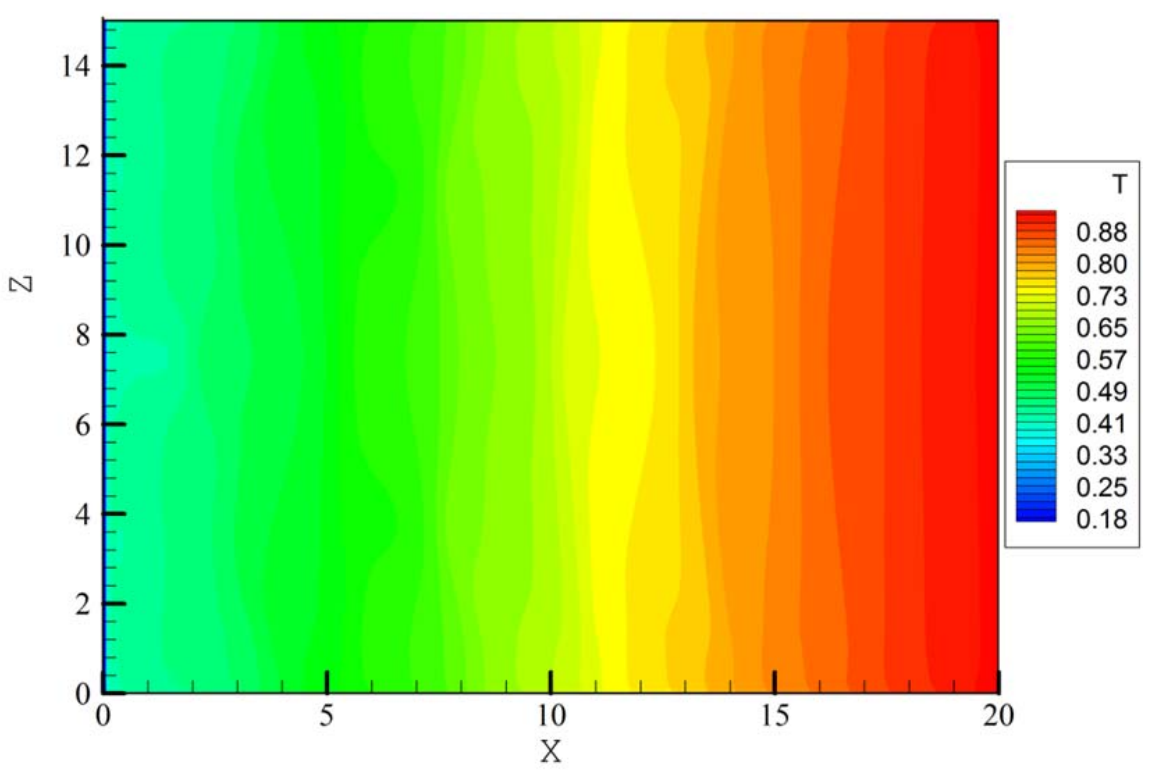

b)

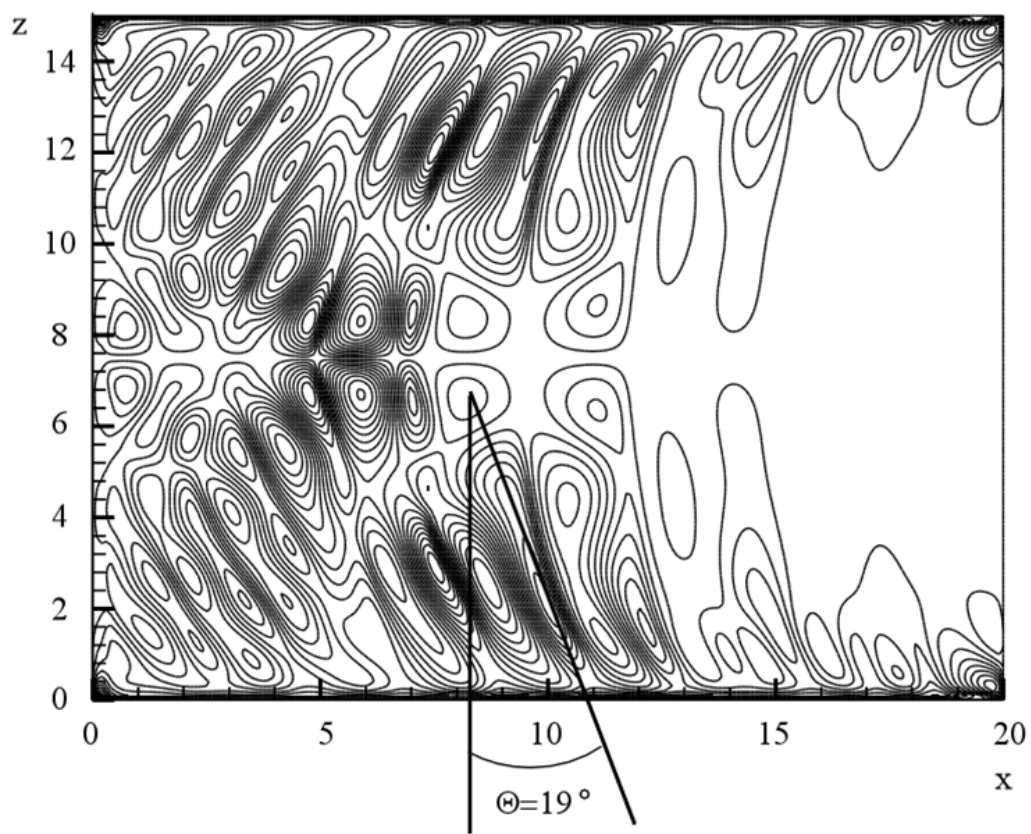

c)

Figure 4: Structure of the thermofluid-dynamic field in a 3D layer of SCN (succinonitrile) with conducting conditions on the bottom wall, isothermal walls at $\mathrm{x}=0$ and $\mathrm{x}=20$, adiabatic surface and adiabatic walls at $\mathrm{z}=0$ and $\mathrm{z}=15\left(\mathrm{~A}_{\mathrm{x}}=20, \mathrm{~A}_{\mathrm{z}}=15, \mathrm{Pr}=23, \mathrm{Ma}=3 \times 10^{4}, \mathrm{t}=2\right)$ : a) isosurfaces (snapshot) of the vertical component of velocity revealing the presence of transverse rolls with slightly distorted shapes, which travel from the cold side towards the hot side, b) snapshot of surface temperature distribution, c) distribution of the longitudinal component of velocity (w) on the free interface revealing the inclination angle of travelling waves with respect to the basic flow. 


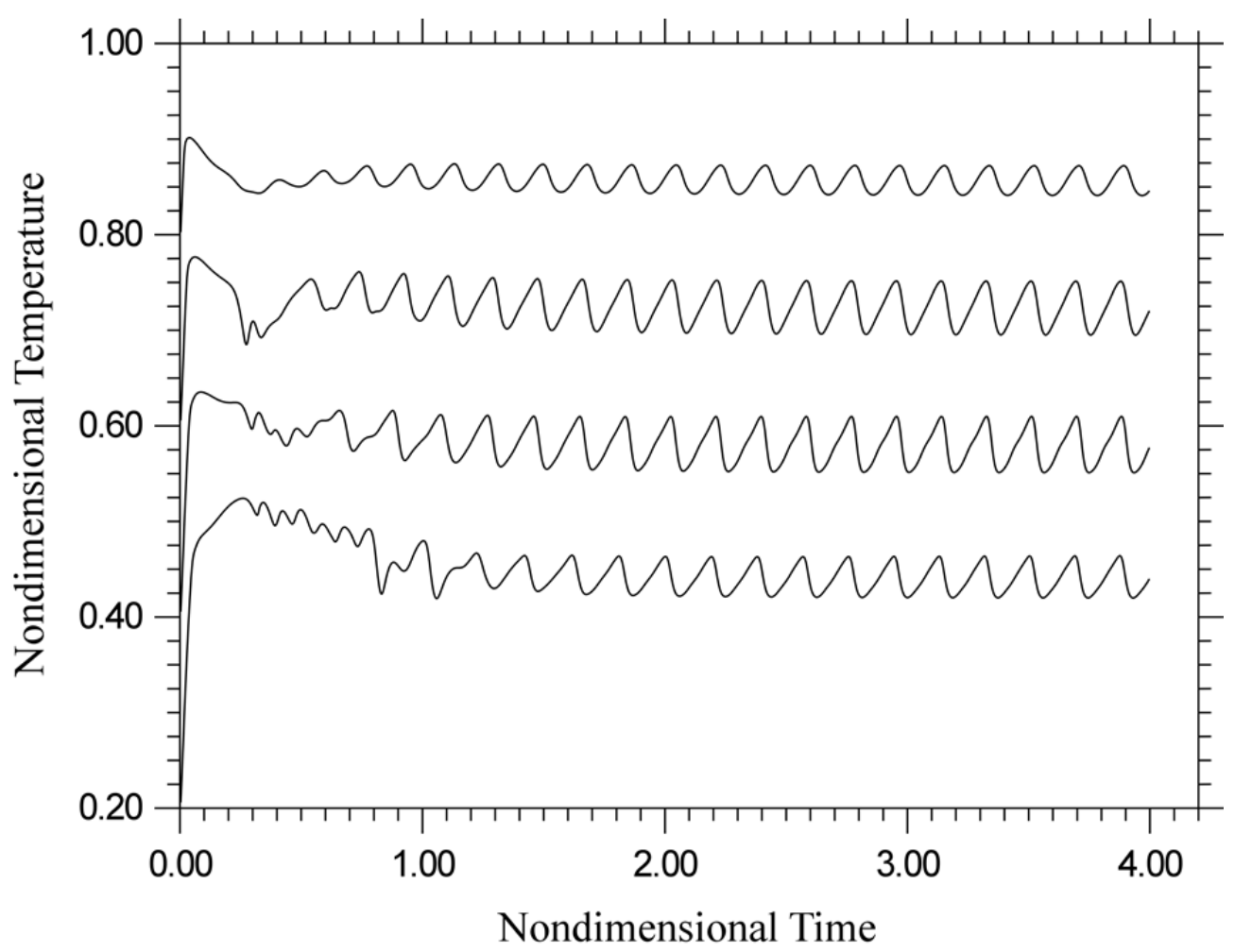

Figure 5: Nondimensional temperature as a function of time at four locations evenly spaced along the horizontal extension of the layer ( $2 \mathrm{D}$ simulation, $\mathrm{NaNO}_{3}, \mathrm{Pr}=8, \mathrm{Ma}=2 \times 10^{4}, \mathrm{~T}_{\mathrm{Hot}}=319{ }^{\circ} \mathrm{C}, \mathrm{T}_{\text {cold }}$ $=308^{\circ} \mathrm{C}, \Omega_{\mathrm{HTW}}=33.2$ ).

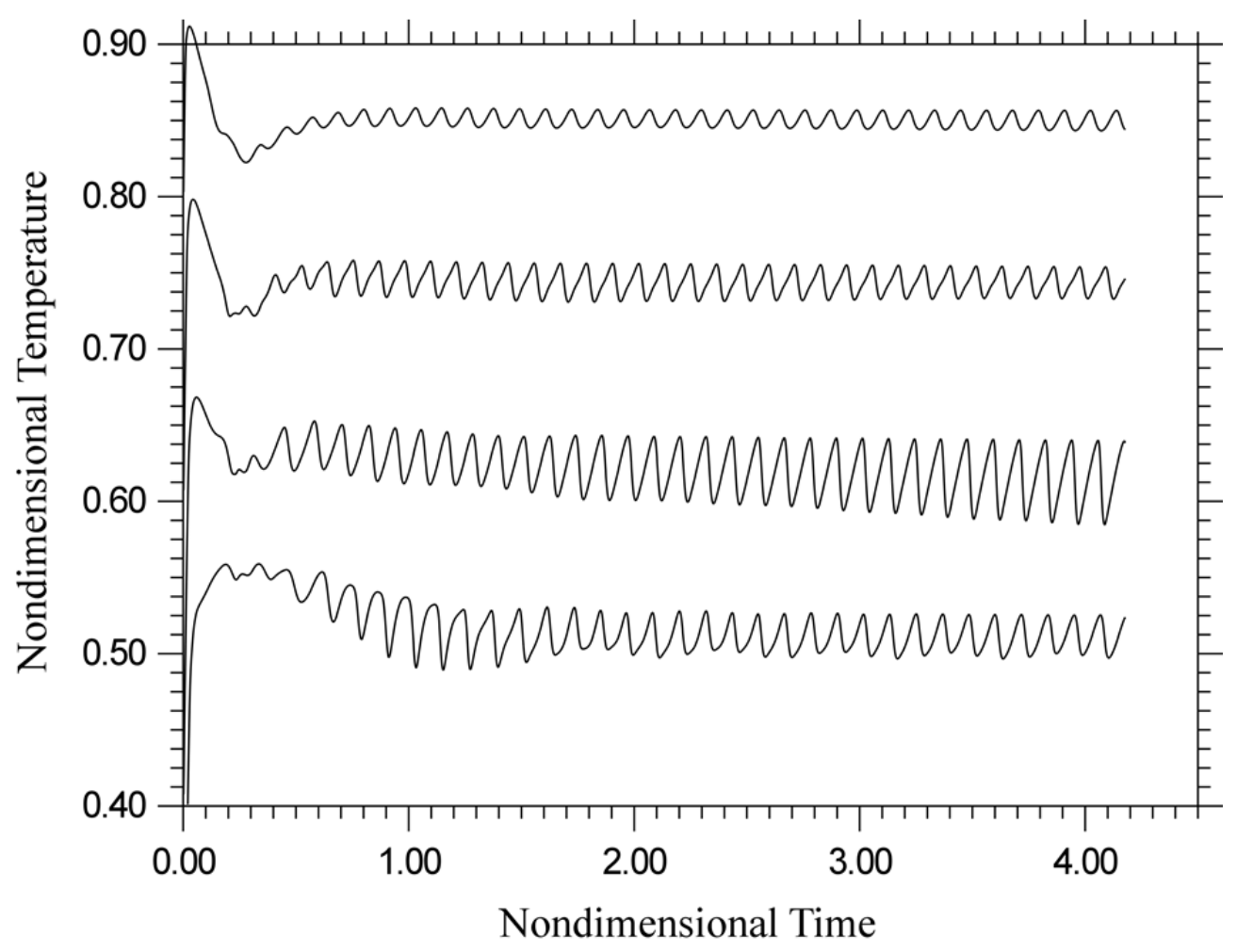

Figure 6: Nondimensional temperature as a function of time at four locations evenly spaced along the horizontal extension of the layer ( $2 \mathrm{D}$ simulation, succinonitrile, $\mathrm{Pr}=23, \mathrm{Ma}=3 \times 10^{4}, \mathrm{~T}_{\mathrm{Hot}}=66.9$ ${ }^{\circ} \mathrm{C}, \mathrm{T}_{\text {cold }}=58.6{ }^{\circ} \mathrm{C}, \Omega_{\mathrm{HTW}}=54.8$ ). 
Along these lines, Figs. 5 and 6 show the non-dimensional temperature signals as measured by four numerical probes $\left(\mathrm{T}_{\mathrm{i}}, \mathrm{i}=1,2,3,4\right)$ located in proximity of the free liquid-gas interface at four positions evenly spaced along the horizontal extension $\left(\mathrm{x} / \mathrm{A}_{\mathrm{x}}=-0.3,-0.1,0.1,0.3\right.$, respectively as shown in Fig. 1b). The aforementioned decay of the disturbance while it approaches the hot side is clearly witnessed by the distribution of signal amplitude along the horizontal direction (the decrease in amplitude of the signal measured by thermocouples can be clearly seen, especially by comparing $\mathrm{T}_{2}$ with $\mathrm{T}_{3}$ and $\mathrm{T}_{4}$ ).

\subsection{Solidifying liquid layer}

Starting at the instant $t^{*}$, corresponding to the last value displayed at the right side of Fig. 5 and 6, respectively, a cooling ramp has been applied on the cold side, as specified in Sect. 2.5, to induce solidification of the melt while keeping fixed the temperature of the hot wall. The following ramps, in particular, have been considered $\mathrm{k}=0.1,0.01$ and $0.001{ }^{\circ} \mathrm{C} \mathrm{s}^{-1}$ for both substances, whereas the final temperature to be established on the cold wall has been fixed to $\mathrm{T}_{\text {liq }}-2 \Delta \mathrm{T}$ (which gives a final value $\mathrm{T}_{\text {cold }}=286{ }^{\circ} \mathrm{C}$ and $\mathrm{T}_{\text {cold }}=42{ }^{\circ} \mathrm{C}$ for sodium nitrate and succinonitrile, respectively).

It should be mentioned that, though distinct values of the ramping rates (differing in the related orders of magnitude) have been expressly selected in order to assess the sensitivity of the considered processes to the degree of unsteadiness introduced by the temperature variation at the boundary, all such processes should be regarded as examples of circumstances for which the solidification occurs at a constant value of the Marangoni number (as defined by eq. (8)).

Indeed, with the initial assumption of temperature on the cold wall fixed to $\mathrm{T}_{\text {liq }}$ for $\mathrm{t} \leq \mathrm{t}^{*}$ (let us recall for the convenience of the reader that, by definition, $\mathrm{T}_{\text {liq }}$ is the minimum constant temperature at which the material is still in a liquid state), a decrease in temperature at this wall for $\mathrm{t}>\mathrm{t}^{*}$ cannot change the effective temperature difference across the liquid. Such a temperature difference, from a purely mathematical point of view, would read $T_{\text {hot }}-T_{\text {front }}$, where $T_{\text {front }}$ is the "average" temperature in the mushy region [namely $\left(\mathrm{T}_{\text {sol }}+\mathrm{T}_{\text {liq }}\right) / 2$ ]. As $\mathrm{T}_{\text {sol }} \cong \mathrm{T}_{\text {liq }}$, however, for simplicity, in the present work it is computed as $\Delta \mathrm{T} \cong\left(\mathrm{T}_{\text {hot }}-\mathrm{T}_{\text {liq }}\right)$ (this representation making immediately clear that though the liquid domain undergoes a shrinkage in time due to the progressive transformation of melt into solid, the difference $\Delta \mathrm{T}$ will retain its initial value during the overall solidification process, i.e. $\Delta \mathrm{T}=11^{\circ} \mathrm{C}$ and $8.3{ }^{\circ} \mathrm{C}$ for sodium nitrate and succinonitrile, respectively).

Given such a premise, and before becoming deeply involved in the elaboration of a comprehensive discussion encapsulating both solidification and fluid mechanical aspects, in the next sections, first we concentrate on the dynamics of the crystallization front and its morphological evolution; then the related changes in terms of waveforms and spectral content of the supercritical flows for the different conditions considered (type of substance and ramping rate used for the solidification process) are discussed at a later stage. 


\section{$\underline{\text { 3.2.1 Crystal morphological evolution and related multicellular pattern }}$}

As shown in Figs. 7-9 and 10-12 a feature common to all cases is the inclination displayed by the advancing solidification front (the solid-liquid interface - SLI), which is inclined against the normal direction and is curved in the growth direction due to Marangoni convection.

The key to understanding this trend lies in considering that the solidification front shape and its position are modulated by the flow. Since the surface flow carries hot liquid from the hot side of the container, accordingly, the upper part of the layer is hotter than the lower one (a generic point at the free liquid/gas surface has a temperature higher than that of other points located at the same horizontal coordinate below the free surface). This causes the inclination of the SLI.

Despite such similarity, however, the changes in time of the effective angle of inclination and especially the velocity of the advancing solidification front do depend on the considered conditions (cooling rate). This can be immediately appreciated by taking a look at Figs. 13-15 where the position of three characteristic points along the crystal front ( $\mathrm{x}_{\text {bottom, }} \mathrm{x}_{\text {intermediate }}$ and $\mathrm{x}_{\text {top }}$ corresponding to $\phi=1 / 2$ for $y=-1 / 2, y=0$ and $y=1 / 2$, respectively) has been plotted as function of time, and Fig. 16, expressly showing the inclination angle $(\varphi)$ with respect to the horizontal wall $[\varphi$ computed as $\left.\tan ^{-1}\left(1 / 2\left(\mathrm{x}_{\text {average }}-\mathrm{x}_{\text {bottom }}\right)^{-1}\right)\right]$. In particular, it can be seen that a decrease in the cooling rate from $\mathrm{k}=0.1$ to $\mathrm{k}=0.01$ determines a decrease in the inclination of the front. By contrast, (as expected) an increase in the cooling rate determines an increase in the horizontal extension of the crystal especially in the initial stages of evolution. 


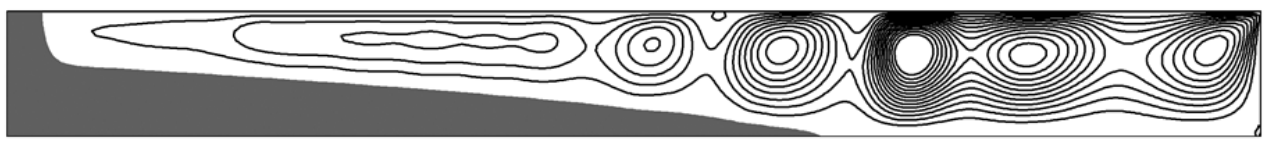

a)

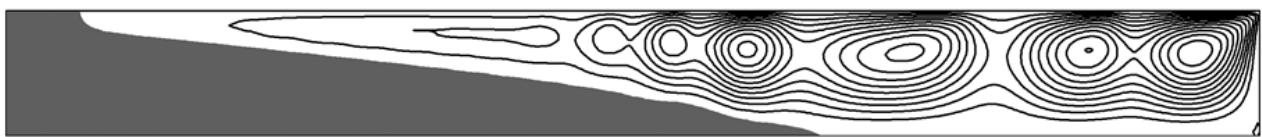

b)

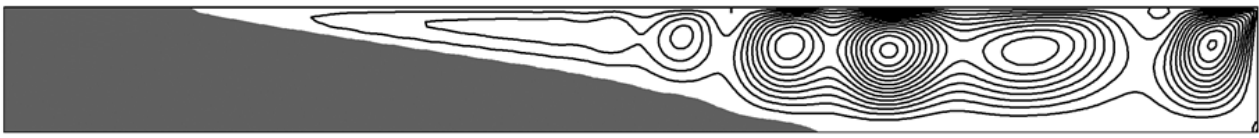

c)

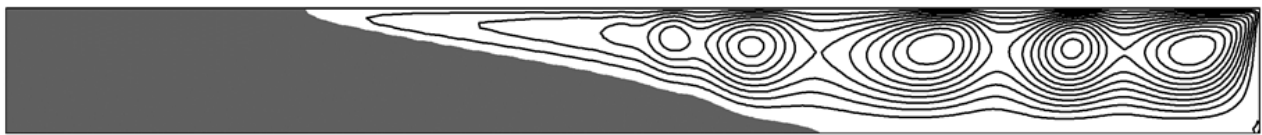

d)

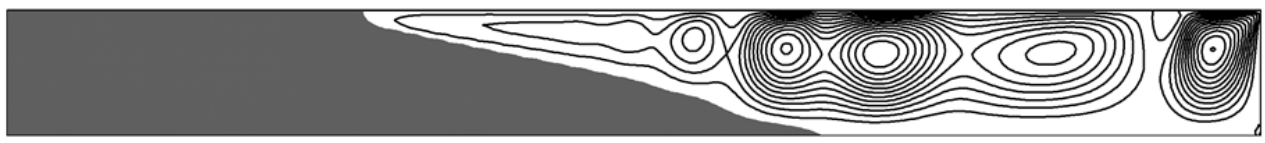

e)

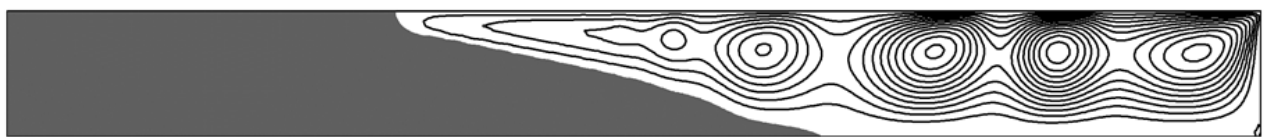

f)

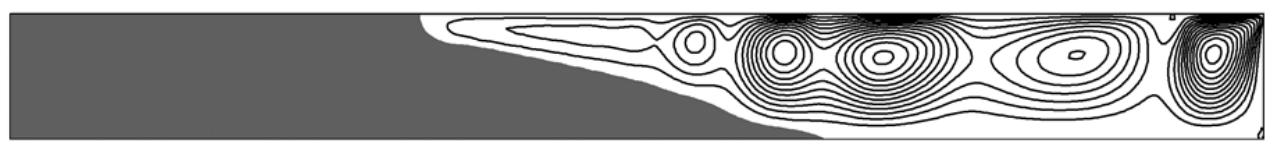

g)

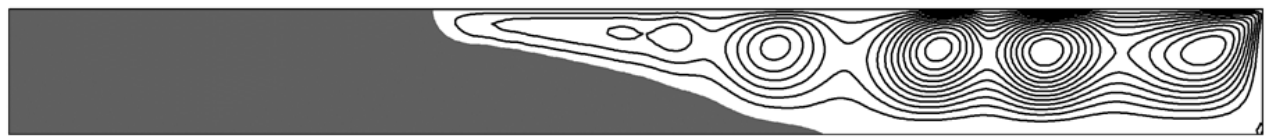

h)

Figure 7: Snapshots of velocity field and solid mass distribution as a function of time $\left(\mathrm{NaNO}_{3}\right.$, $\operatorname{Pr}=8, \mathrm{Ma}=2 \times 10^{4}, \mathrm{~T}_{\mathrm{Hot}}=319{ }^{\circ} \mathrm{C},\left(\mathrm{T}_{\text {cold }}\right)_{\text {fin }}=286{ }^{\circ} \mathrm{C}$, ramping rate $\mathrm{k}=0.1{ }^{\circ} \mathrm{C} / \mathrm{s}, \psi_{\max }=60, \Delta \psi \cong 4.3$, the nondimensional time interval between two consecutive frames is $\cong 1.08$ corresponding to approx. $685 \mathrm{~s})$. 


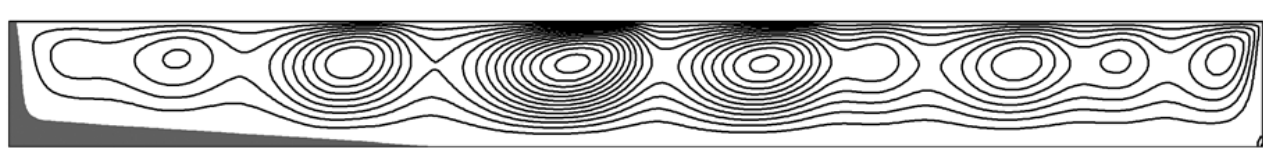

a)

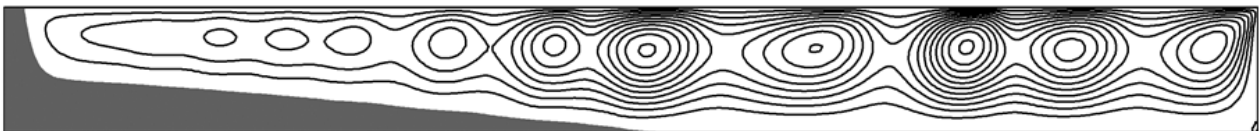

b)

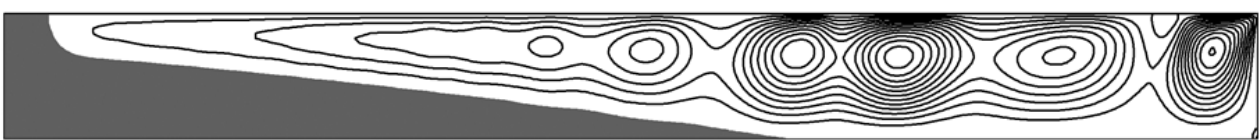

c)

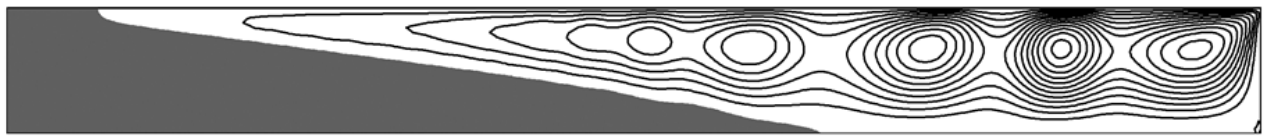

d)

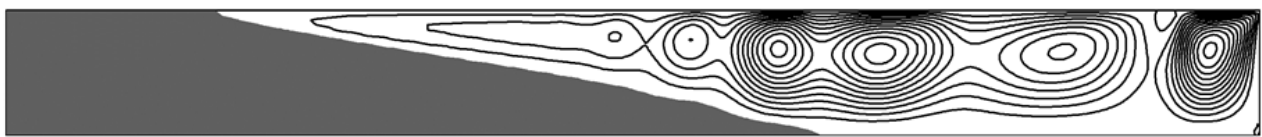

e)

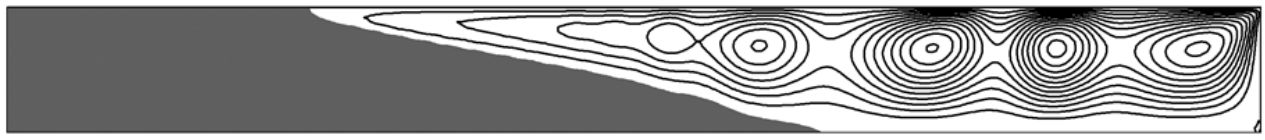

f)

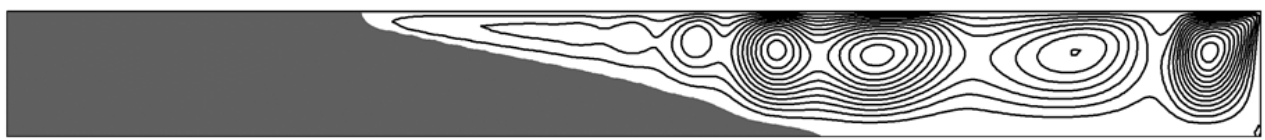

g)

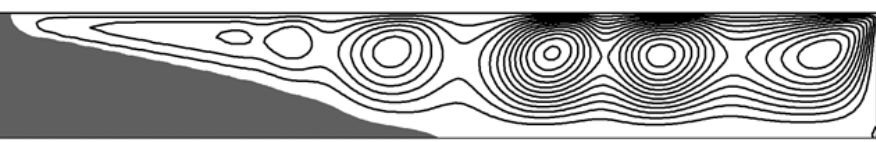

h)

Figure 8: Snapshots of velocity field and solid mass distribution as a function of time (NaNO3, $\mathrm{Pr}=8, \mathrm{Ma}=2 \times 10^{4}, \mathrm{~T}_{\mathrm{Hot}}=319^{\circ} \mathrm{C},\left(\mathrm{T}_{\text {cold }}\right)_{\text {fin }}=286{ }^{\circ} \mathrm{C}$, ramping rate $\mathrm{k}=0.01{ }^{\circ} \mathrm{C} / \mathrm{s}, \psi_{\max }=60, \Delta \psi \cong 4.3$, the nondimensional time interval between two consecutive frames is $\cong 1.08$ corresponding to approx. $685 \mathrm{~s})$. 


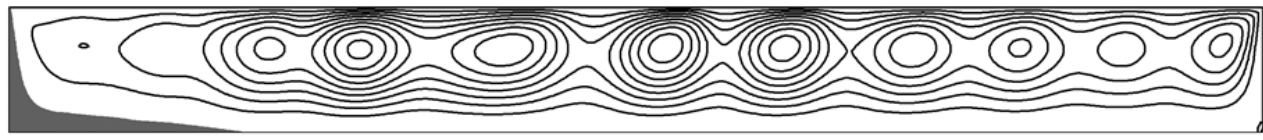

a)

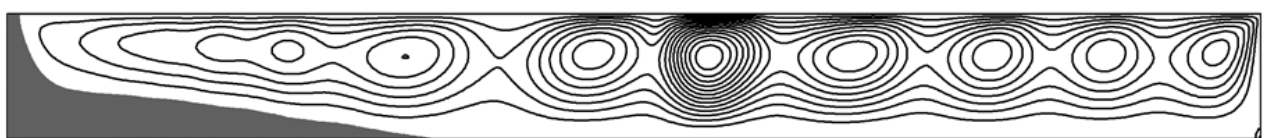

b)

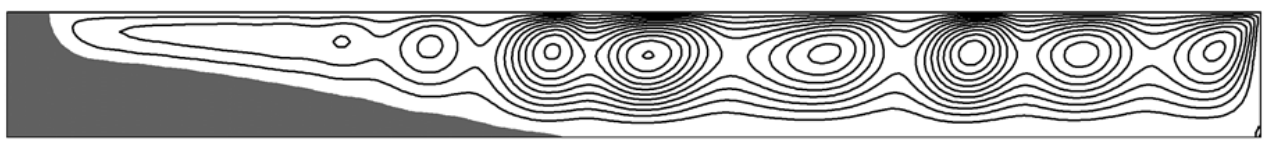

c)

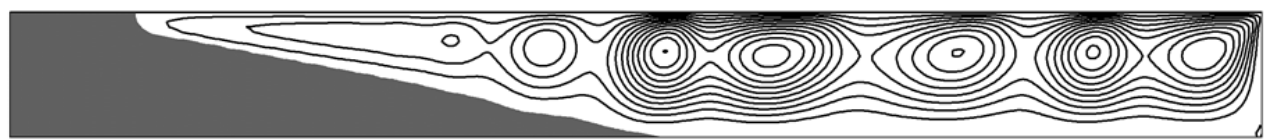

d)

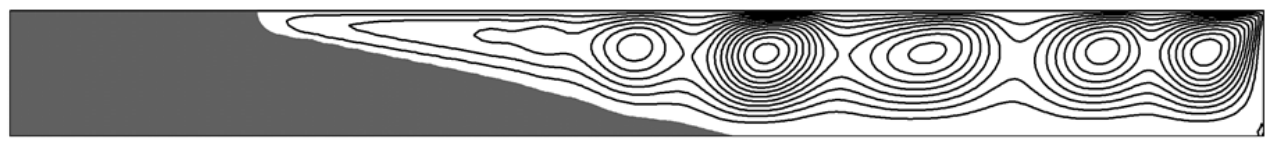

e)
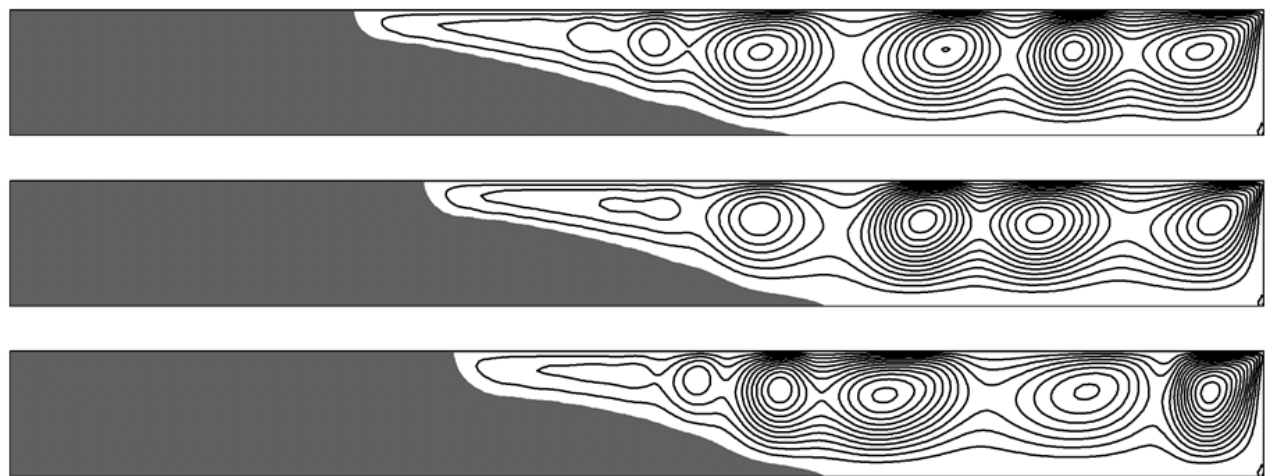

Figure 9: Snapshots of velocity field and solid mass distribution as a function of time (NaNO3, $\operatorname{Pr}=8, \mathrm{Ma}=2 \times 10^{4}, \mathrm{~T}_{\text {Hot }}=319{ }^{\circ} \mathrm{C},\left(\mathrm{T}_{\text {cold }}\right)_{\text {fin }}=286{ }^{\circ} \mathrm{C}$, ramping rate $\mathrm{k}=0.001{ }^{\circ} \mathrm{C} / \mathrm{s}, \psi_{\max }=60, \Delta \psi \cong 4.3$, the nondimensional time interval between two consecutive frames is $\cong 5.19$ corresponding to approx. $3284 \mathrm{~s})$. 


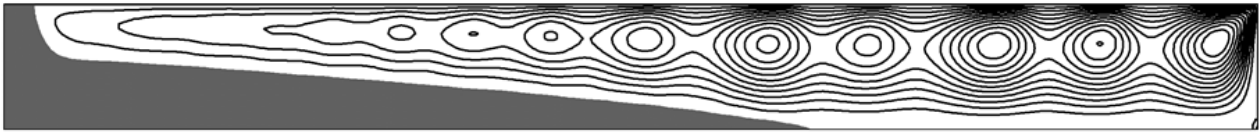

a)

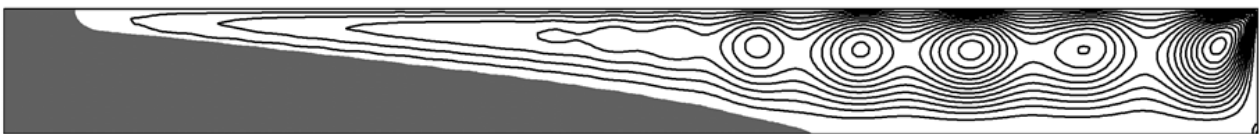

b)

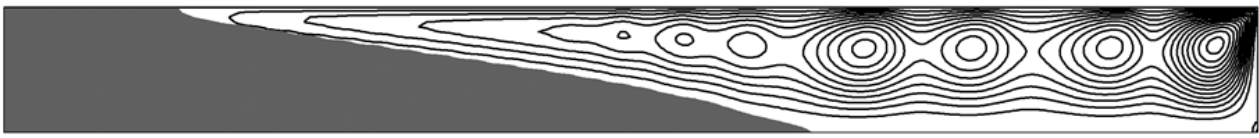

c)

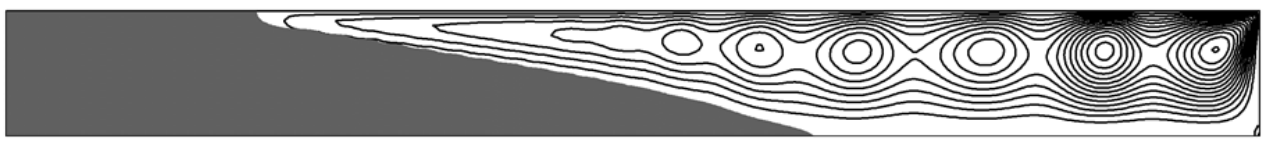

d)

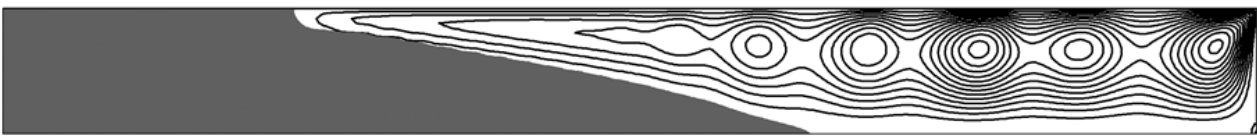

e)
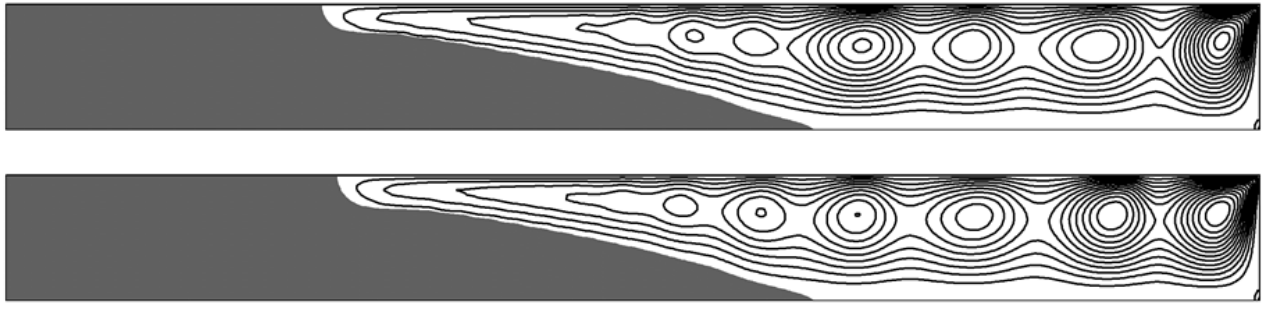

g)

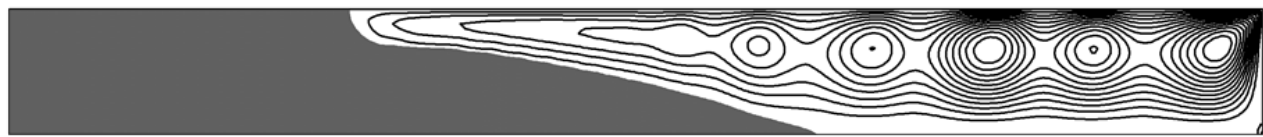

h)

Figure 10: Snapshots of velocity field and solid mass distribution as a function of time (Succinonitrile, $\mathrm{Pr}=23, \mathrm{Ma}=3 \times 10^{4}, \mathrm{~T}_{\mathrm{Hot}}=66.9{ }^{\circ} \mathrm{C},\left(\mathrm{T}_{\text {cold }}\right)_{\text {fin }}=42{ }^{\circ} \mathrm{C}$, ramping rate $\mathrm{k}=0.1{ }^{\circ} \mathrm{C} / \mathrm{s}$, $\psi_{\max }=60, \Delta \psi \cong 4.3$, the nondimensional time interval between two consecutive frames is $\cong 0.5$ corresponding to approx. $444 \mathrm{~s}$ ). 


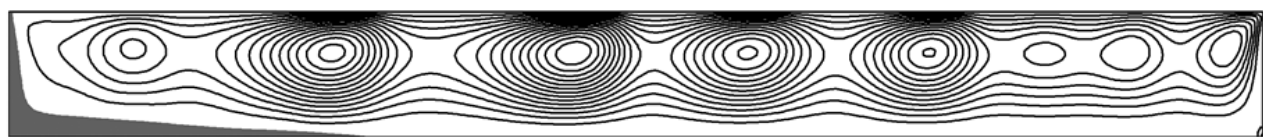

a)

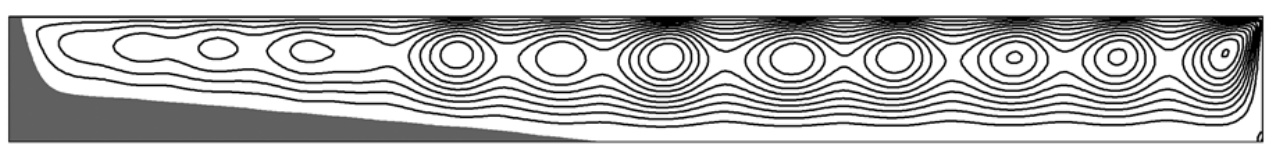

b)

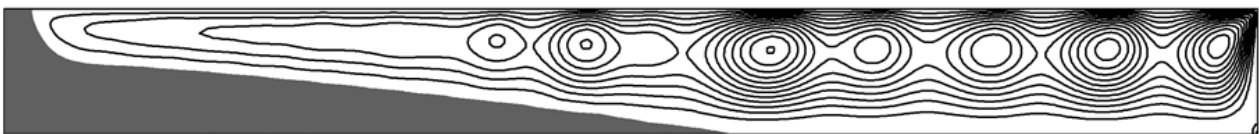

c)

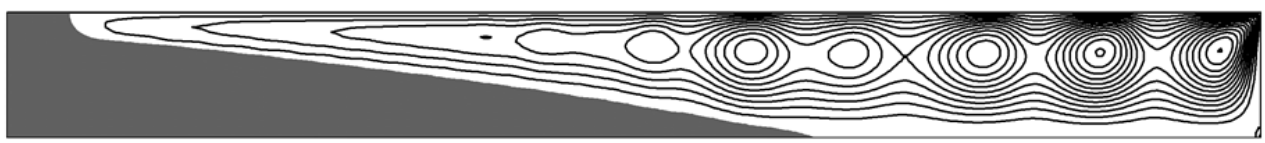

d)

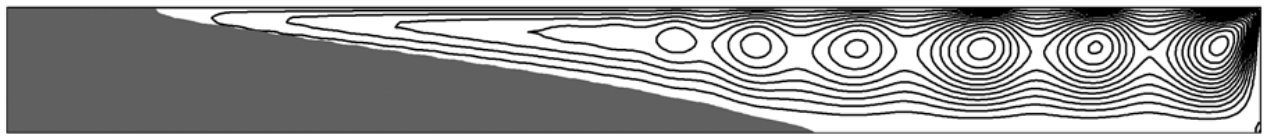

e)
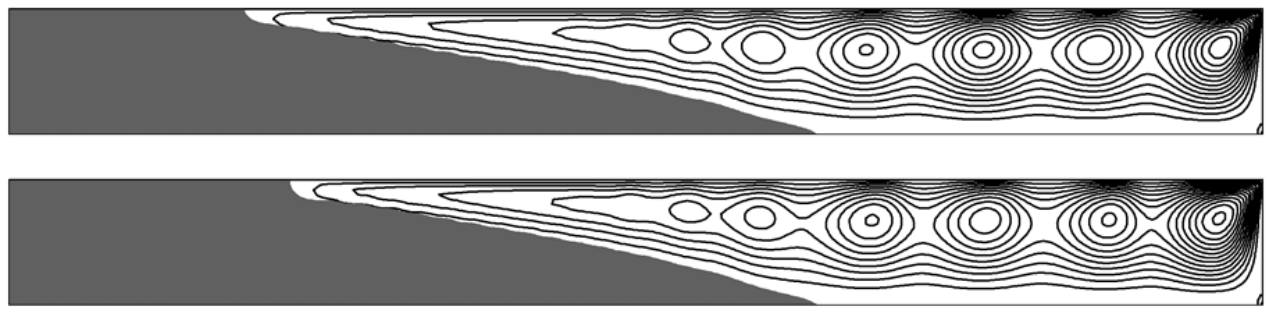

g)

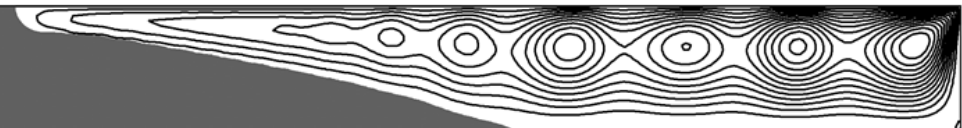

h)

Figure 11: Snapshots of velocity field and solid mass distribution as a function of time (Succinonitrile, $\mathrm{Pr}=23, \mathrm{Ma}=3 \times 10^{4}, \mathrm{~T}_{\mathrm{Hot}}=66.9{ }^{\circ} \mathrm{C}$, $\left(\mathrm{T}_{\text {cold }}\right)_{\text {fin }}=42{ }^{\circ} \mathrm{C}$, ramping rate $\mathrm{k}=0.01{ }^{\circ} \mathrm{C} / \mathrm{s}$, $\psi_{\max }=60, \Delta \psi \cong 4.3$, the nondimensional time interval between two consecutive frames is $\cong 0.5$ corresponding to approx. $444 \mathrm{~s}$ ) 


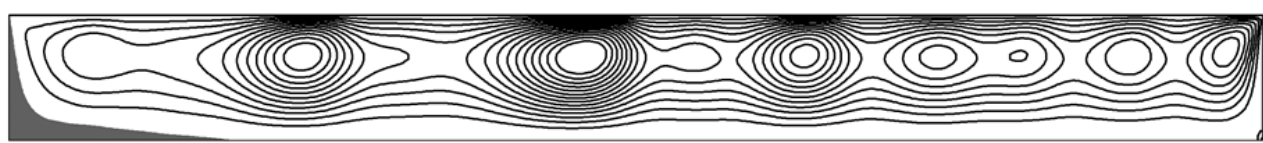

a)

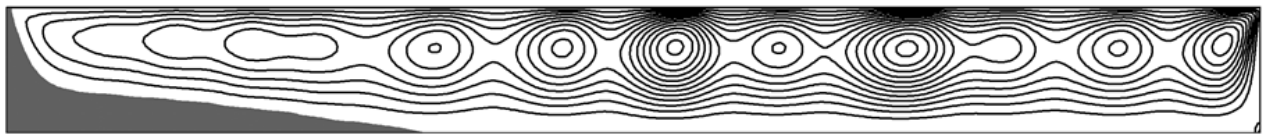

b)

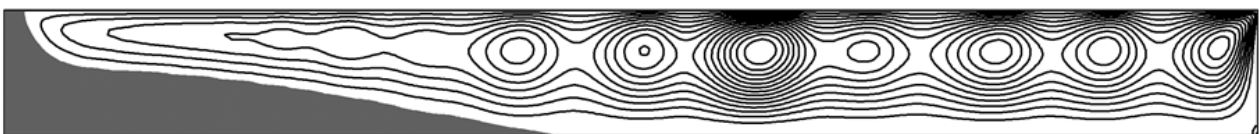

c)

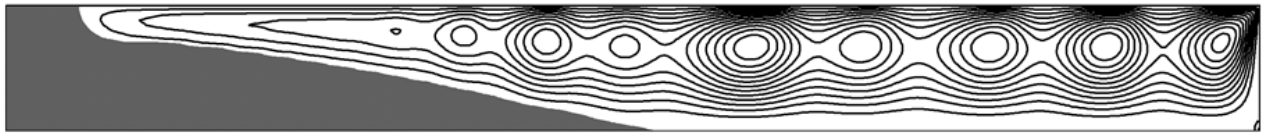

d)

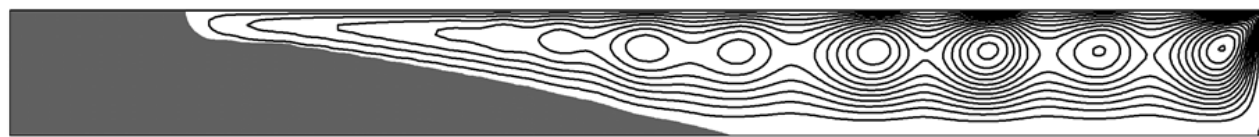

e)
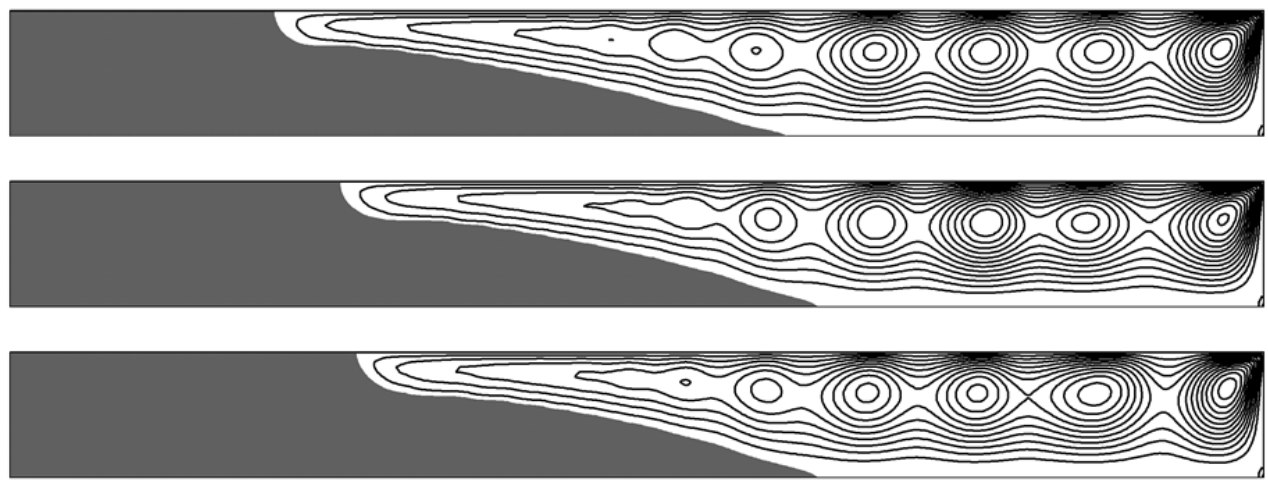

h)

Figure 12: Snapshots of velocity field and solid mass distribution as a function of time (Succinonitrile, $\mathrm{Pr}=23, \mathrm{Ma}=3 \times 10^{4}, \mathrm{~T}_{\mathrm{Hot}}=66.9{ }^{\circ} \mathrm{C},\left(\mathrm{T}_{\text {cold }}\right)_{\text {fin }}=42{ }^{\circ} \mathrm{C}$, ramping rate $\mathrm{k}=0.001{ }^{\circ} \mathrm{C} / \mathrm{s}$, $\psi_{\max }=60, \Delta \psi \cong 4.3$, the nondimensional time interval between two consecutive frames is $\cong 2.84$ corresponding to approx. $2514 \mathrm{~s}$ )

Interestingly, all figures show that for the present circumstances (fixed temperature on the hot side and constant value of the Marangoni number) the system tends towards an asymptotic solution with the solid-melt interface located at an equilibrium position in the middle of the layer.

Leaving aside for a while specific morphological details and related arguments, Figs. 7-9 and 10-12 also provide very interesting insights into the changes experienced by the flow structure, i.e. the patterning behaviour.

As expected, the initial multicellular pattern undergoes shrinkage in the horizontal direction due to the progressive expansion (growth) of the solid phase. Most interestingly, however, in the initial stages of solidification, an increase in the number of rolls present in the liquid phase can be noticed (up to $\mathrm{N}=10$ ). This unexpected effect (most likely induced by the surface fluid acceleration resulting from the application of the temperature ramp) can be clearly seen in all the figures by looking at frames (a), (b) or (c). 
As time passes, however, the contraction of the space effectively available for fluid flow (see Figs. 13-15) causes a decrease in the number of rolls present on average in the container. Such a number decreases progressively from the initial value to a final value that depends only on the considered type of melt $(\mathrm{N}=4$ for $\operatorname{Pr}=8$ and $\mathrm{N}=5$ for $\mathrm{Pr}=23$ ). Due to the almost horizontal configuration of the crystal shape, however, some smaller-size cells survive in the liquid region bounded from below by the crystal. In line with earlier findings available for the case of travelling waves developing in obstructed cavities (e.g., in the presence of a backward-facing or forward-facing step on the bottom wall, [42]), these satellite cells do participate in the overall oscillatory behaviour (although their size is much smaller than the other rolls located in the unobstructed part of the cavity).
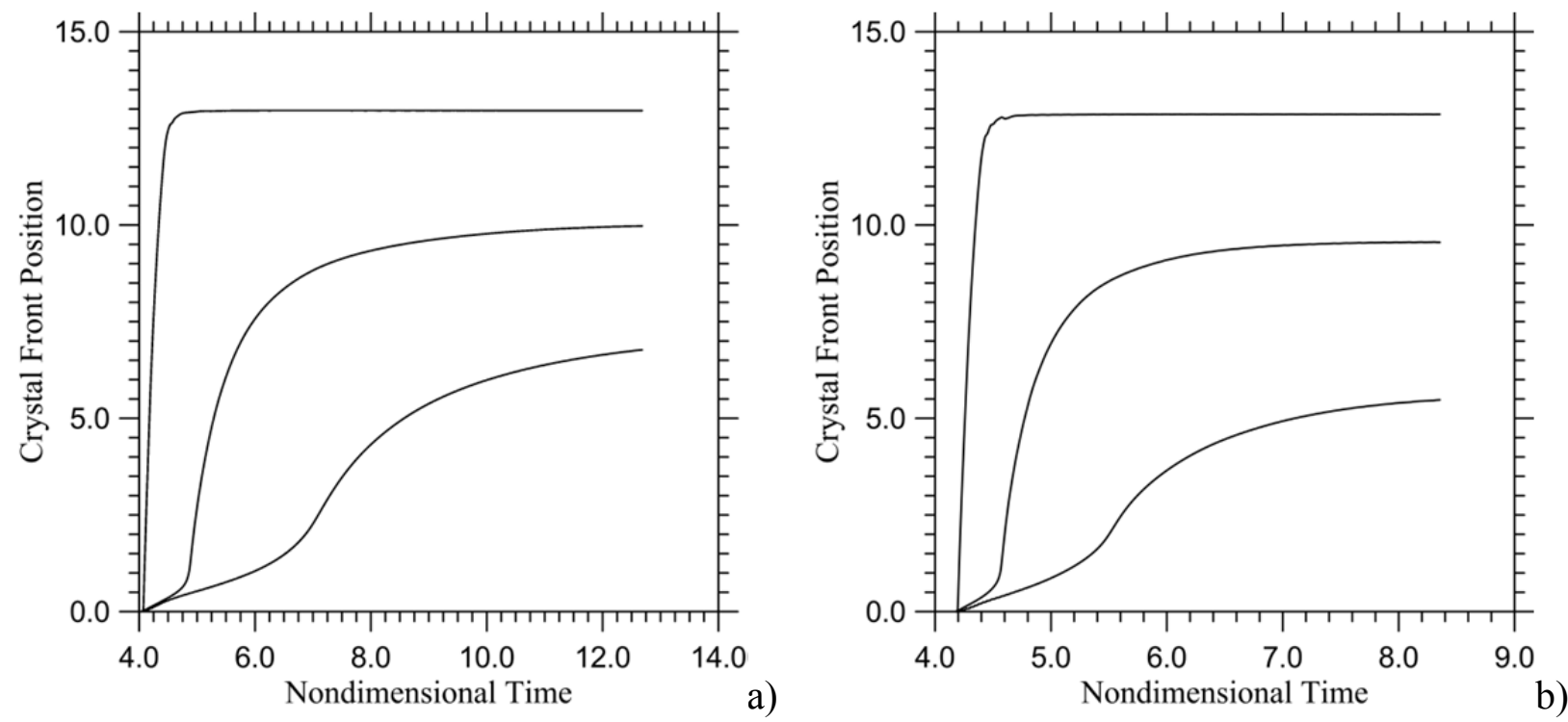

Figure 13: Distance of crystal front from the cold wall (for $y=-1 / 2, y=0$ and $y=1 / 2$ ) as a function of time for $\mathrm{k}=0.1^{\circ} \mathrm{C} / \mathrm{s}$ : a) $\mathrm{NaNO} 3$, b) $\mathrm{SCN}$.
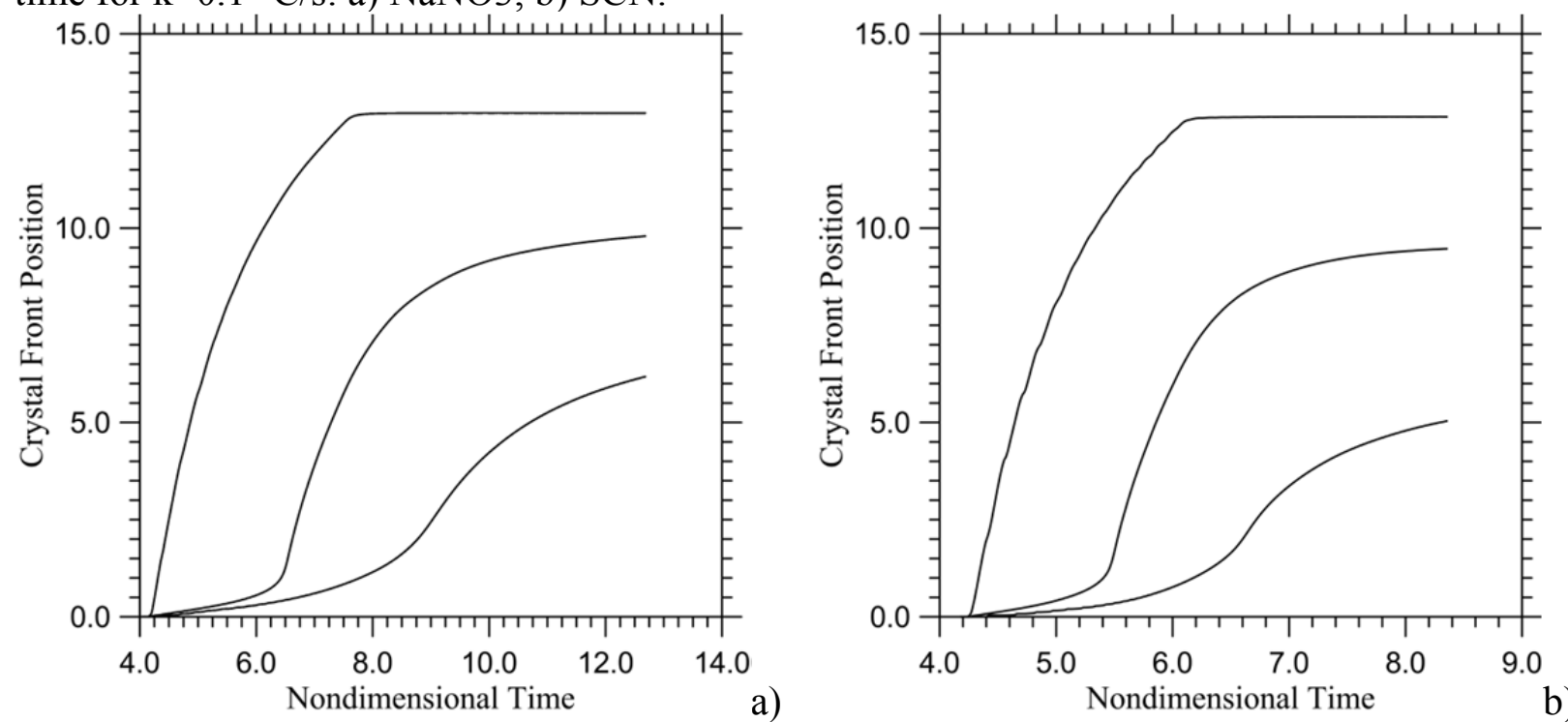

Figure 14: Distance of crystal front from the cold wall (for $y=-1 / 2, y=0$ and $y=1 / 2$ ) as a function of time for $\mathrm{k}=0.01{ }^{\circ} \mathrm{C} / \mathrm{s}$ : a) $\left.\mathrm{NaNO} 3, \mathrm{~b}\right) \mathrm{SCN}$. 

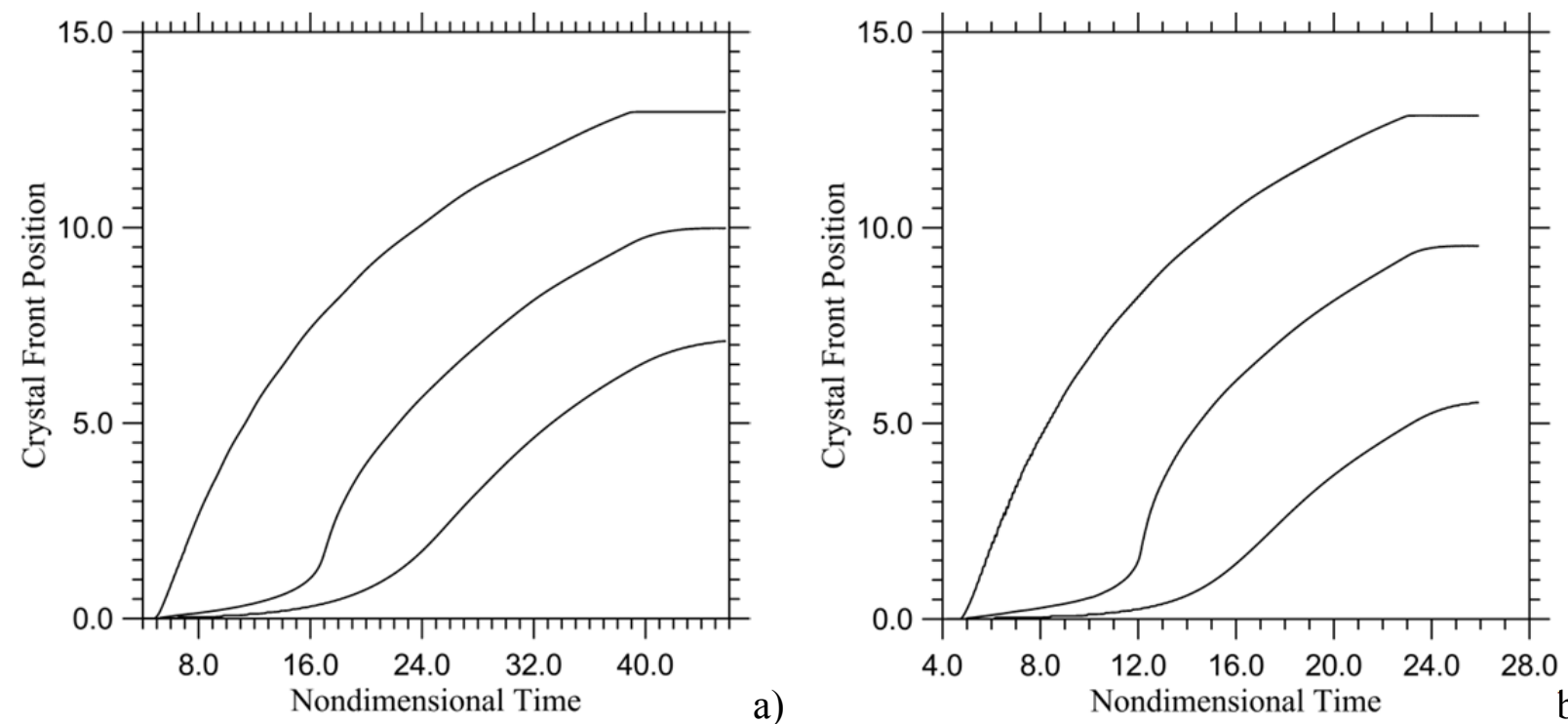

Figure 15: Distance of crystal front from the cold wall (for $y=-1 / 2, y=0$ and $y=1 / 2$ ) as a function of time for $\mathrm{k}=0.001^{\circ} \mathrm{C} / \mathrm{s}$ : a) $\mathrm{NaNO} 3$, b) $\mathrm{SCN}$.
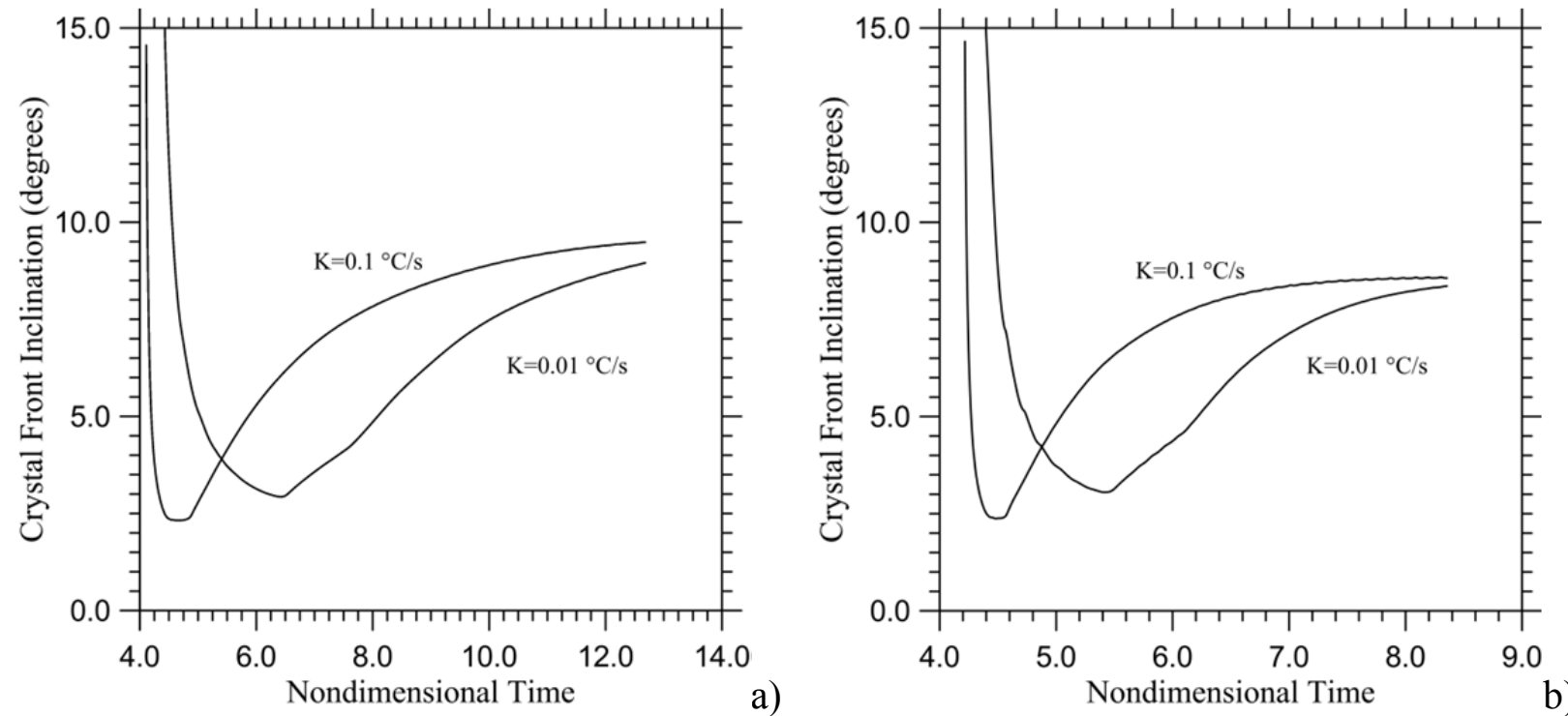

Figure 16: Inclination angle $\varphi$ of the crystal front with respect to the horizontal direction as a function of time: a) NaNO3, b) SCN.

\subsubsection{Thermocouple outputs and frequency trends}

Having finished a sketch of the patterning behaviour for both the solid and liquid phases, we now turn to re-examine the results in terms of frequency trends and related implications.

To avoid duplications with respect to what has been already illustrated in the preceding text, most conveniently, we focus on the spectral content of the temperature field in conjunction with earlier arguments given for the evolution of the solid phase. 


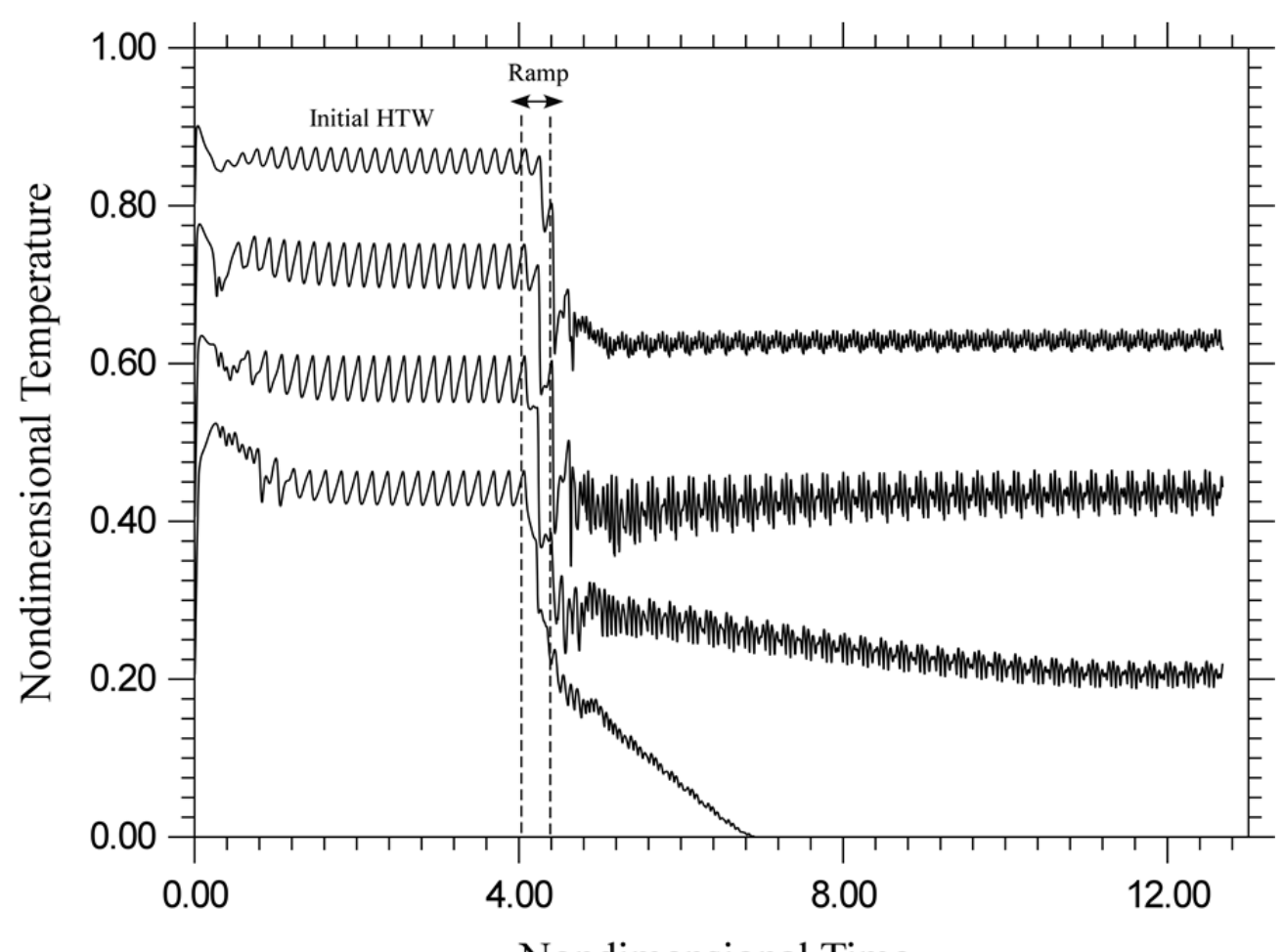

a)

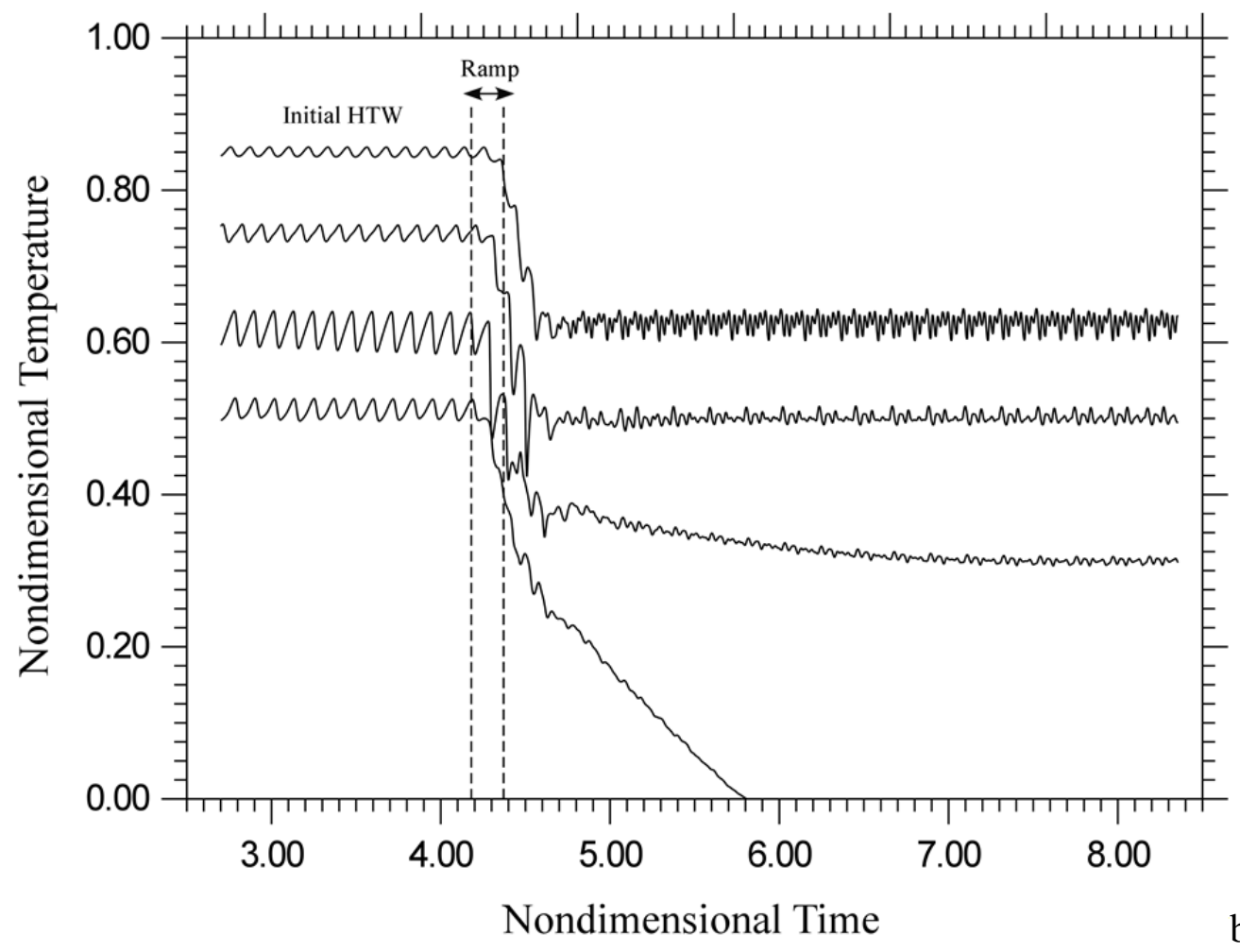

Figure 17: Nondimensional temperature as a function of time at four locations evenly spaced along the horizontal extension of the layer for $\mathrm{k}=0.1{ }^{\circ} \mathrm{C} / \mathrm{s}$ : a) $\operatorname{Pr}=8\left(\mathrm{NaNO}_{3}\right), \mathrm{Ma}=2 \times 10^{4}, \mathrm{~T}_{\mathrm{Hot}}=319{ }^{\circ} \mathrm{C}$, $\left(\mathrm{T}_{\text {cold }}\right)_{\text {fin }}=286^{\circ} \mathrm{C}$; b) $\mathrm{Pr}=23$ (Succinonitrile), $\mathrm{Ma}=3 \times 10^{4}, \mathrm{~T}_{\mathrm{Hot}}=66.9^{\circ} \mathrm{C},\left(\mathrm{T}_{\text {cold }}\right)_{\text {fin }}=42^{\circ} \mathrm{C}$. 


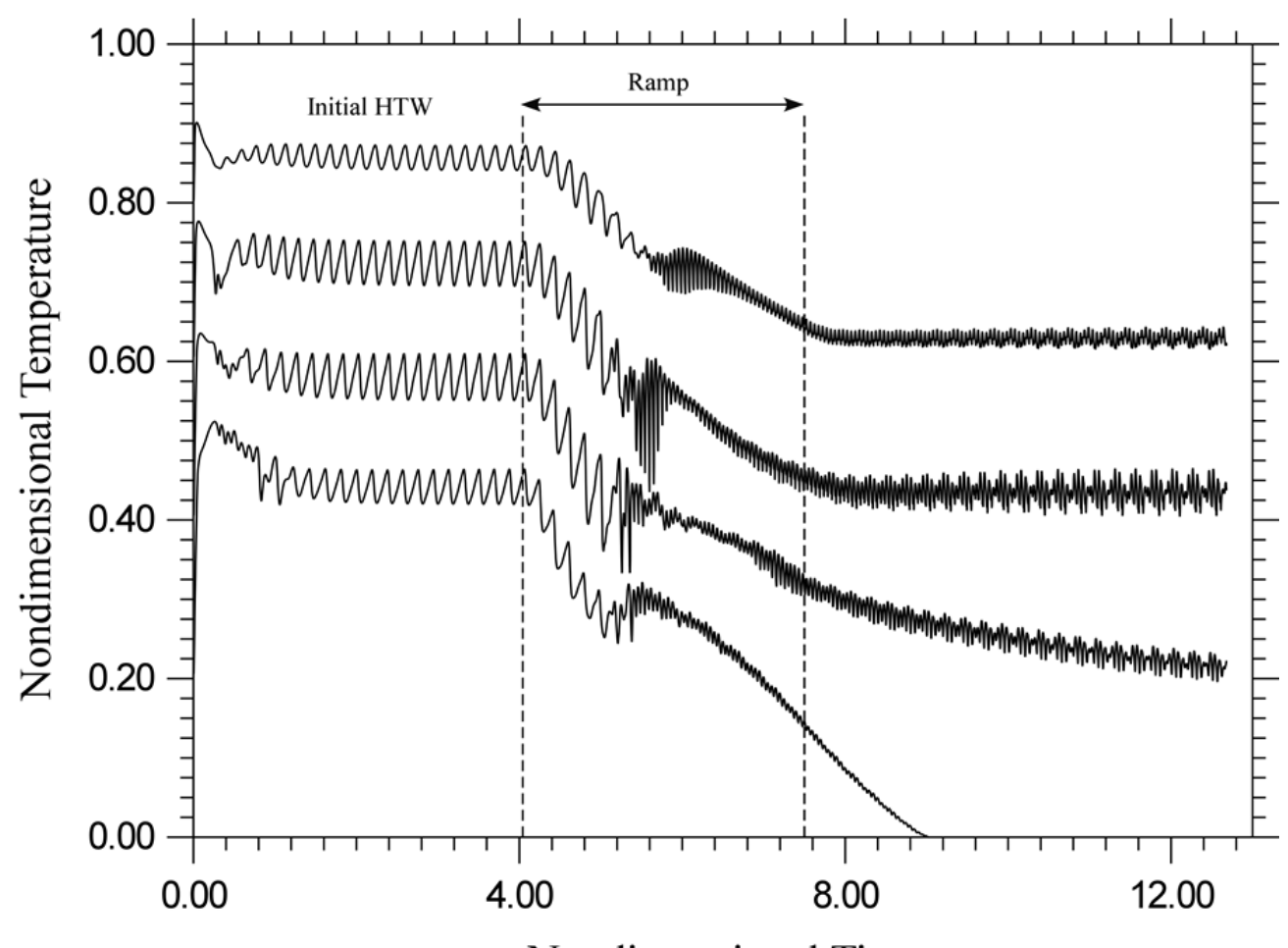

Nondimensional Time

a)

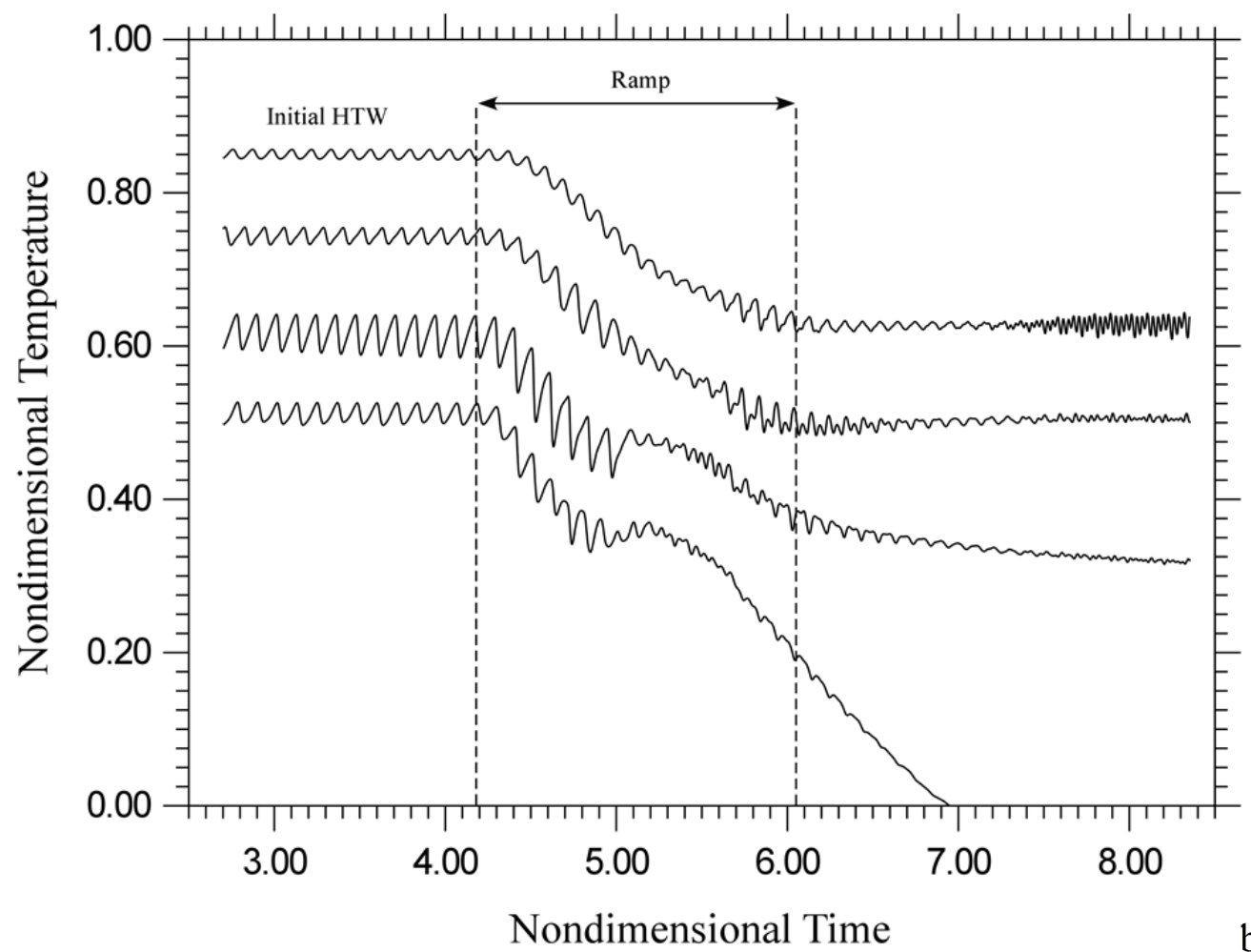

Figure 18: Nondimensional temperature as a function of time at four locations evenly spaced along the horizontal extension of the layer for $\mathrm{k}=0.01{ }^{\circ} \mathrm{C} / \mathrm{s}$ : a) $\mathrm{Pr}=8\left(\mathrm{NaNO}_{3}\right), \mathrm{Ma}=2 \times 10^{4}, \mathrm{~T}_{\mathrm{Hot}}=319^{\circ} \mathrm{C}$, $\left(\mathrm{T}_{\text {cold }}\right)_{\text {fin }}=286{ }^{\circ} \mathrm{C}$; b) Pr=23 (Succinonitrile), $\mathrm{Ma}=3 \times 10^{4}, \mathrm{~T}_{\mathrm{Hot}}=66.9^{\circ} \mathrm{C},\left(\mathrm{T}_{\text {cold }}\right)$ fin $=42^{\circ} \mathrm{C}$. 

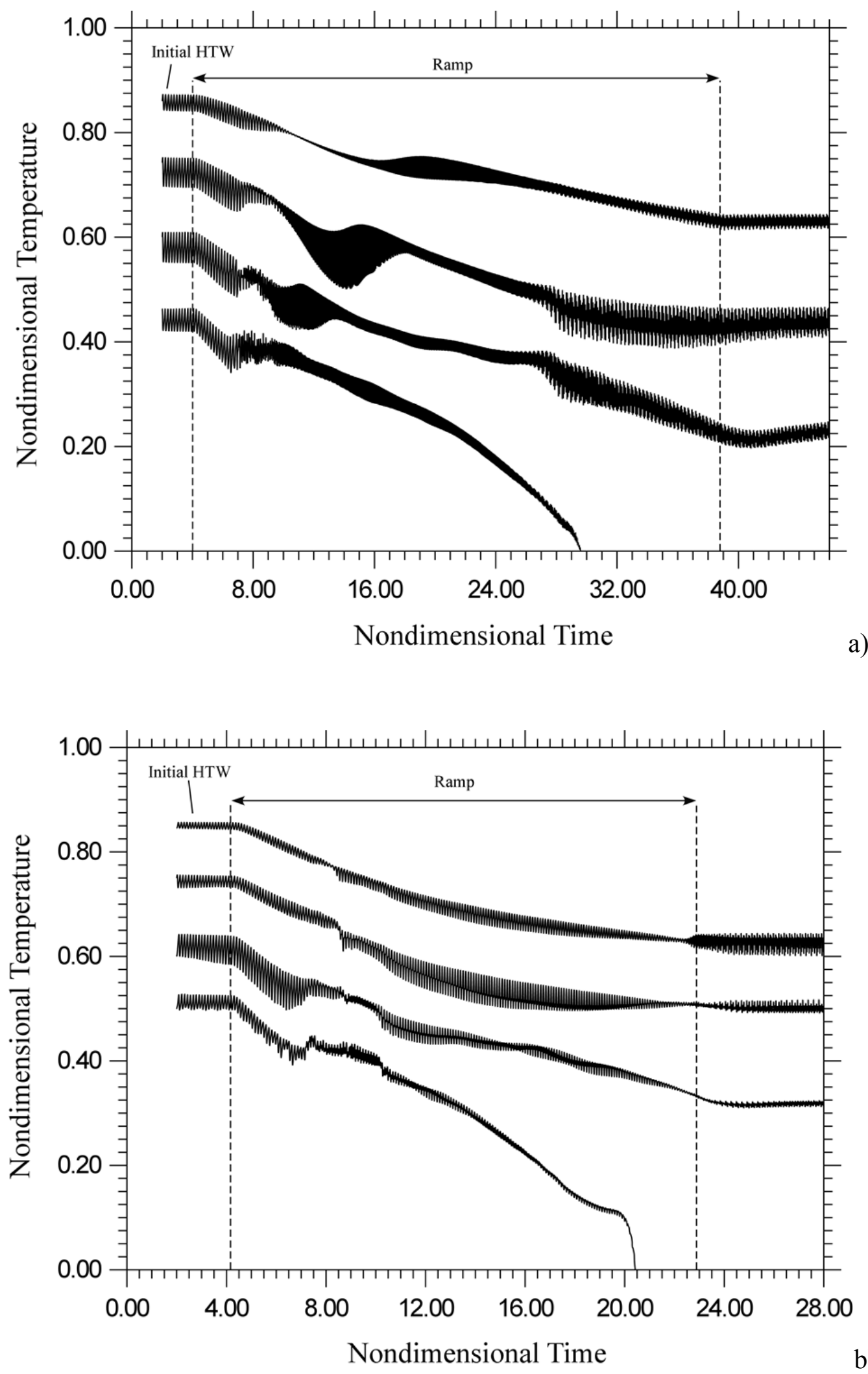

Figure 19: Nondimensional temperature as a function of time at four locations evenly spaced along the horizontal extension of the layer for $\mathrm{k}=0.001{ }^{\circ} \mathrm{C} / \mathrm{s}$ : a) $\mathrm{Pr}=8\left(\mathrm{NaNO}_{3}\right), \mathrm{Ma}=2 \times 10^{4}, \mathrm{~T}_{\mathrm{Hot}}=319^{\circ} \mathrm{C}$, $\left(\mathrm{T}_{\text {cold }}\right)_{\text {fin }}=286^{\circ} \mathrm{C}$; b) $\mathrm{Pr}=23$ (Succinonitrile), $\mathrm{Ma}=3 \times 10^{4}, \mathrm{~T}_{\mathrm{Hot}}=66.9^{\circ} \mathrm{C},\left(\mathrm{T}_{\text {cold }}\right)_{\text {fin }}=42^{\circ} \mathrm{C}$. 
Inspection of Fig. 17 reveals that after a short transient time (corresponding essentially to the application of the cooling ramp on the cold wall) the flow evolves towards a new regime. Indeed, a clear change in the fundamental angular frequency can be observed (from $\Omega_{\mathrm{HTW}} \cong 34.06$ to $\Omega_{1} \cong 26.5$ for $\mathrm{NaNO}_{3}$ and from $\Omega_{\mathrm{HTW}} \cong 54.2$ to $\Omega_{1} \cong 58.3$ for $\mathrm{SCN}$ ). The modifications in terms of temporal behaviour, however, are not limited to a variation of the fundamental frequency. In fact, the frequency spectrum becomes more complex with the emergence of a second much higher frequency $\left(\Omega_{2} \cong 124.7\right.$ for $\mathrm{NaNO}_{3}$ and $\Omega_{2} \cong 117.3$ for $\mathrm{SCN}$ ). Similar behaviours occur for the other considered values of the cooling rate (Figs. 18 and 19, respectively - in practice, the final frequencies do not depend appreciably on the considered cooling rate). The evolution of the frequency spectra with time for the case with $\mathrm{k}=0.001{ }^{\circ} \mathrm{C} / \mathrm{s}$ can be seen in Figs. 20 and 21 for $\mathrm{NaNO}_{3}$ and $\mathrm{SCN}$, respectively. These spectra confirm that, as just discussed above, during the application of the ramp a second frequency is excited so that in the final state is affected by two main frequencies. Interestingly, for the case of $\mathrm{NaNO}_{3}$, a third modulation, is also present with frequency $\Omega_{3}$ equal to the difference of the two other frequencies (this phenomenon is produced by the obvious non-linear interference between the other two oscillatory mechanisms).

As shown in Fig. 22, these oscillatory phenomena also affect (weakly) the crystal shape. Indeed, the lower part of the crystal front (i.e. the signal relating to $\mathrm{x}_{\text {bottom}}$ ) displays a barely appreciable oscillatory behaviour in the asymptotic state (which may indicate periodic solidification and remelting produced by the flow oscillation occurring there). This is in line with the observed structure and oscillatory behaviour of the convection rolls. The intensity and size of these rolls are still relatively large in the region where the crystal front intersects the bottom wall, while they decrease in the region delimited from below by the inclined crystal front and from above by the free surface (which also provides a reasonable justification for the reason why no oscillatory behavior can be detected in the $x_{\text {intermediate }}$ and $x_{\text {top }}$ signals, shown in Figs. 13-15).

It is also worth pointing out that, though (for a fixed value of the Prandtl number) the final value of the temperature difference along the liquid-gas interface is the same for all cases, however, the aforementioned asymptotic behaviour of the system is not completely independent from the cooling rate. This effect can be ascribed to the different stages of evolution taken by the system during the solidification process, which finally produces some appreciable differences in the final shape of the crystal (which in turn result in a different instantaneous shape of the no-slip boundary limiting the flow from the left). 


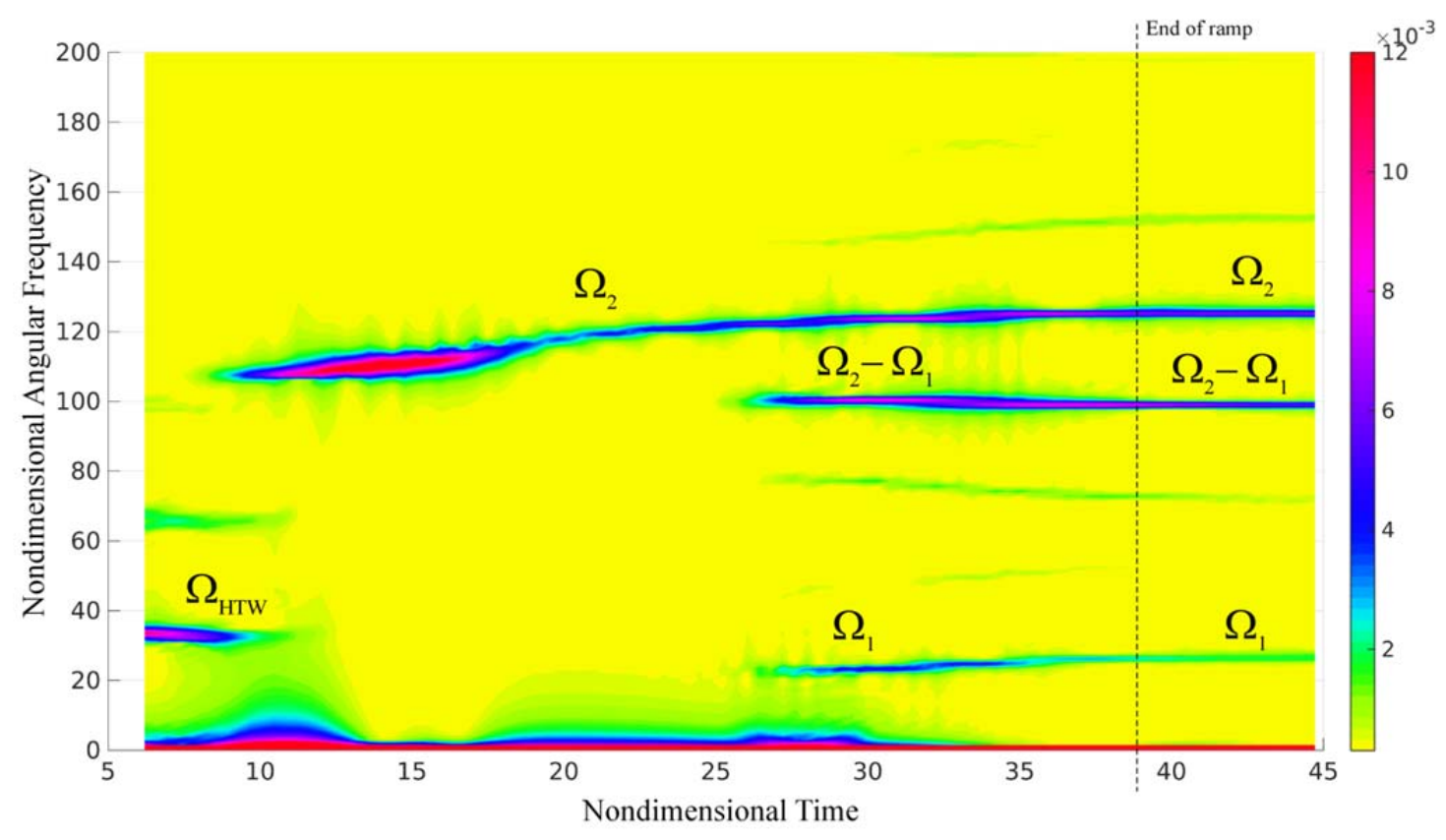

Figure 20: Frequency map for $\mathrm{k}=0.001^{\circ} \mathrm{C} / \mathrm{s}$ and $\mathrm{NaNO}_{3}$.

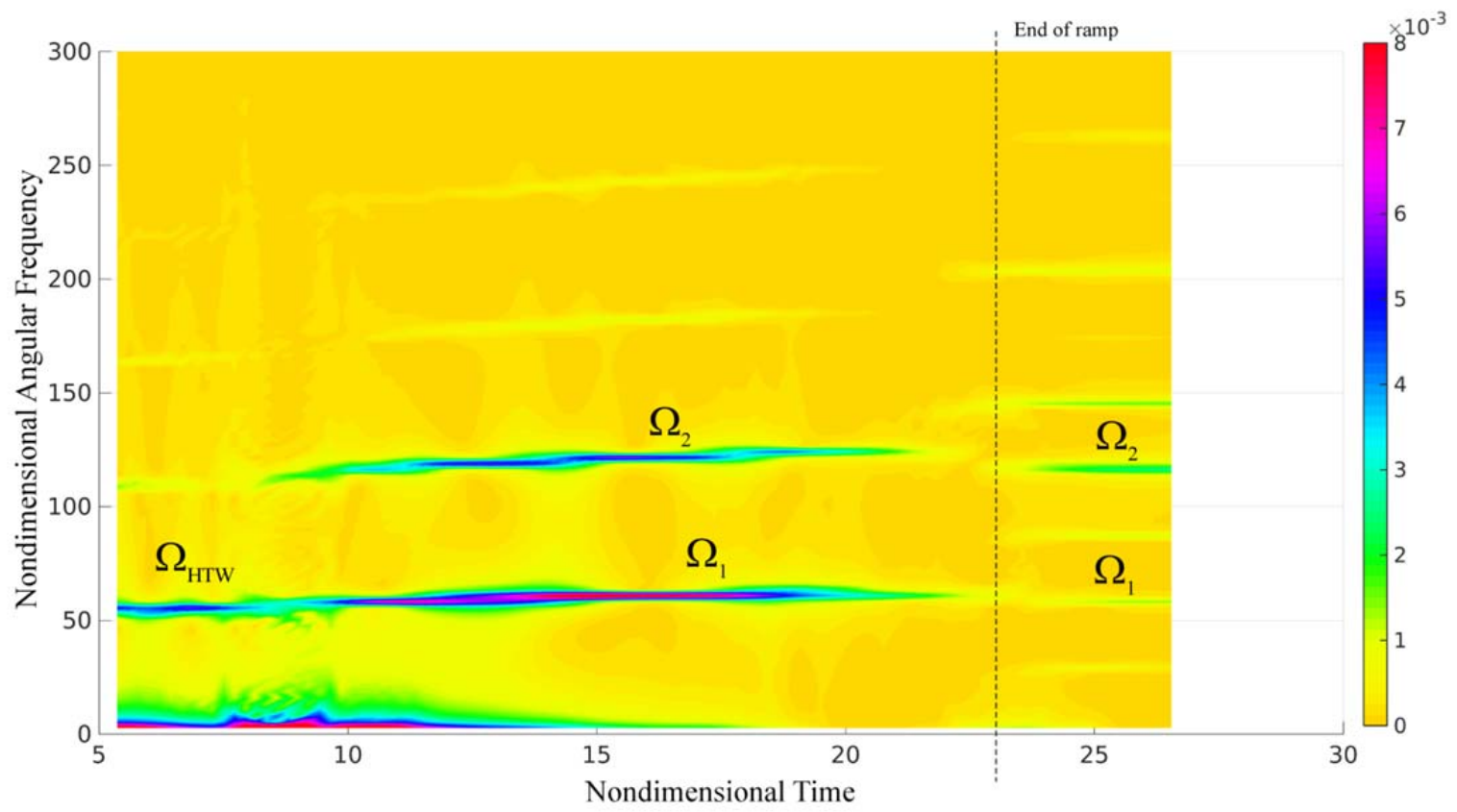

Figure 21: Frequency map for $\mathrm{k}=0.001{ }^{\circ} \mathrm{C} / \mathrm{s}$ and $\mathrm{SCN}$. 

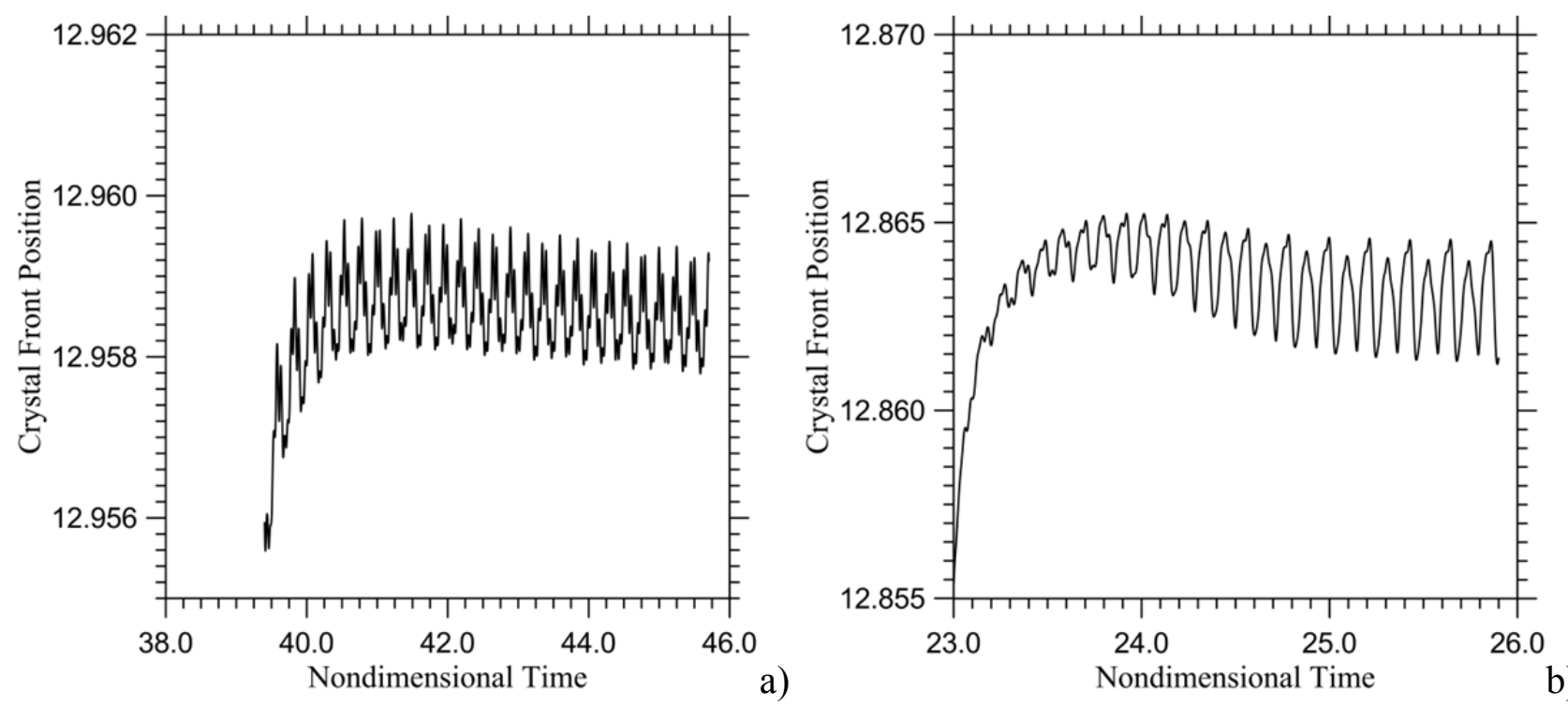

Figure 22: Barely appreciable oscillatory behaviour (with frequency $\Omega_{1}$ ) displayed by the crystal front at $\mathrm{y}=0$ for $\mathrm{k}=0.001^{\circ} \mathrm{C} / \mathrm{s}$ : a) $\mathrm{NaNO} 3$, b) $\mathrm{SCN}$.

Regardless of the minimal differences in the asymptotic behaviours (depending, as just explained, on the specific value assigned to $\mathrm{k}$ ), however, for all cases the significant changes in the spectral content discussed above (with respect to the classical HTW developing in a layer without phase change) can be linked back to corresponding modifications of the visible patterning behaviour.

Most notably, in fact, after the emergence in the spectrum of the $\Omega_{2}$ frequency, the aforementioned property of rolls of being disturbances spreading periodically from the cold side towards the hot side (see Sect 3.1) is no longer a characteristic of the flow. Rather than moving continuously from the cooled side to the heated one, the rolls seem to undergo some back and forth motion around intermediate positions that do not change in time.

In particular, as shown in Fig. 23, a disturbance propagating in the same direction of the surface Marangoni flow can clearly be identified. 


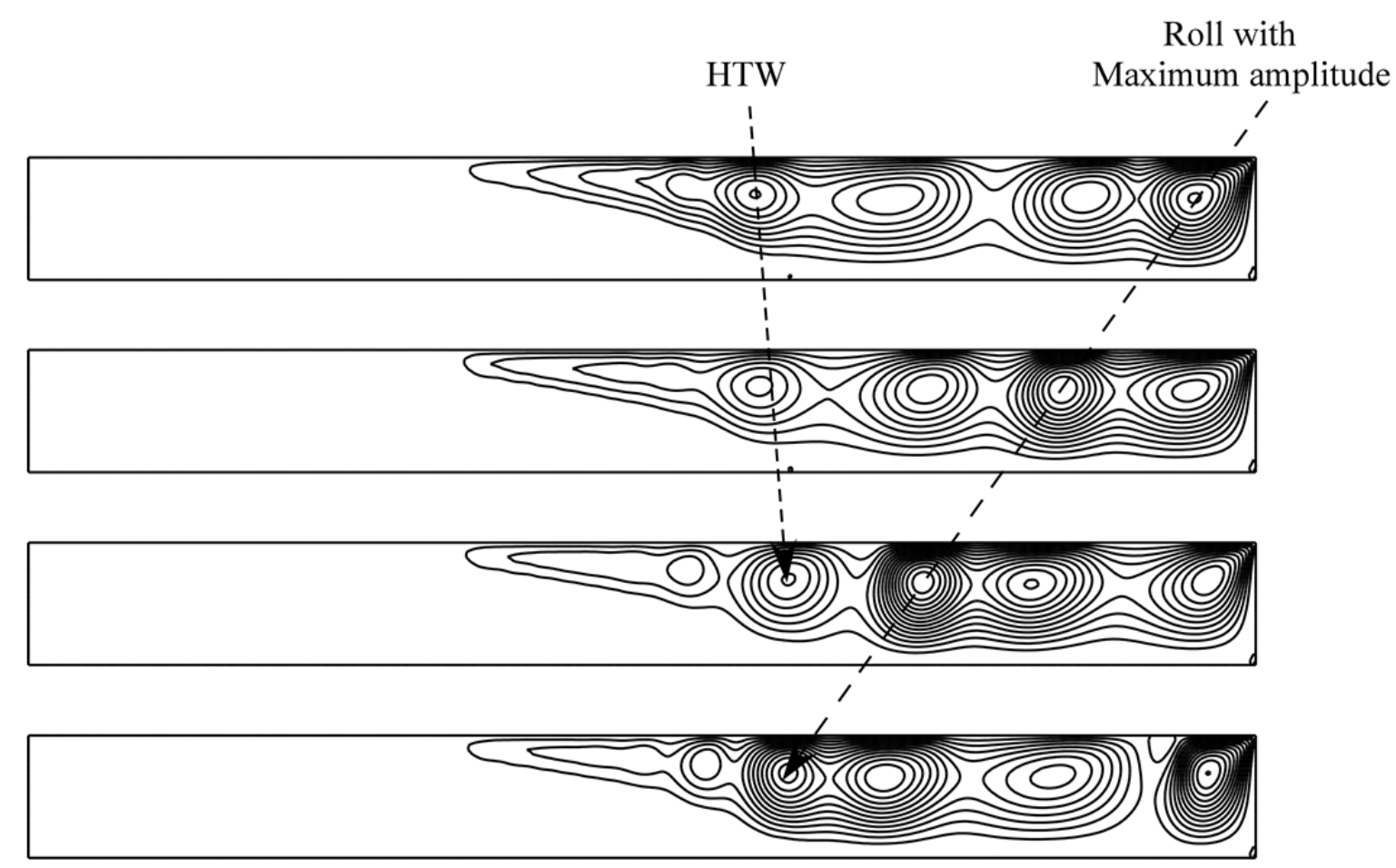

Fig. 23: Snapshots of streamlines at four instants evenly spaced in time during one period of oscillation at the frequency $\Omega_{1}$ for the same conditions shown in Fig. 17a.

This disturbance does not correspond to the physical displacement of a roll, rather it consists of a periodic increase in the roll strength, which now travels in the downstream direction.

In particular, the increase in amplitude originates at the right side roll and, as time passes, it is then transferred to the next roll on the left. Such a process is iterated until the disturbance reaches the solidification front where it dies (Fig. 23, large arrow).

Such observations clearly indicate the presence of an underlying wave travelling in the downstream direction (i.e. i.e. from the hot side to the cold side) at the frequency $\Omega_{2}$ that is much larger than the initial frequency of the HTW $\Omega_{\mathrm{HTW}}$. The essentially pulsating nature of the observed patterns, which behaves globally as a standing wave (the rolls travel continuously neither in the upstream nor in the downstream direction), however, also indicates that some disturbance still propagating in the upstream direction must be present (so that counter-propagating effects can balance to produce a standing-wave-like behaviour). This second disturbance can be identified in the original hydrothermal wave (as witnessed by the proximity of $\Omega_{1}$ to the frequency of the HTW before the application of the cooling ramp). This can also be clearly seen in Fig. 23 where a roll moving at a relatively small angular frequency $\left(\Omega_{1}\right)$ has been indicated (Fig. 23, small arrow). 


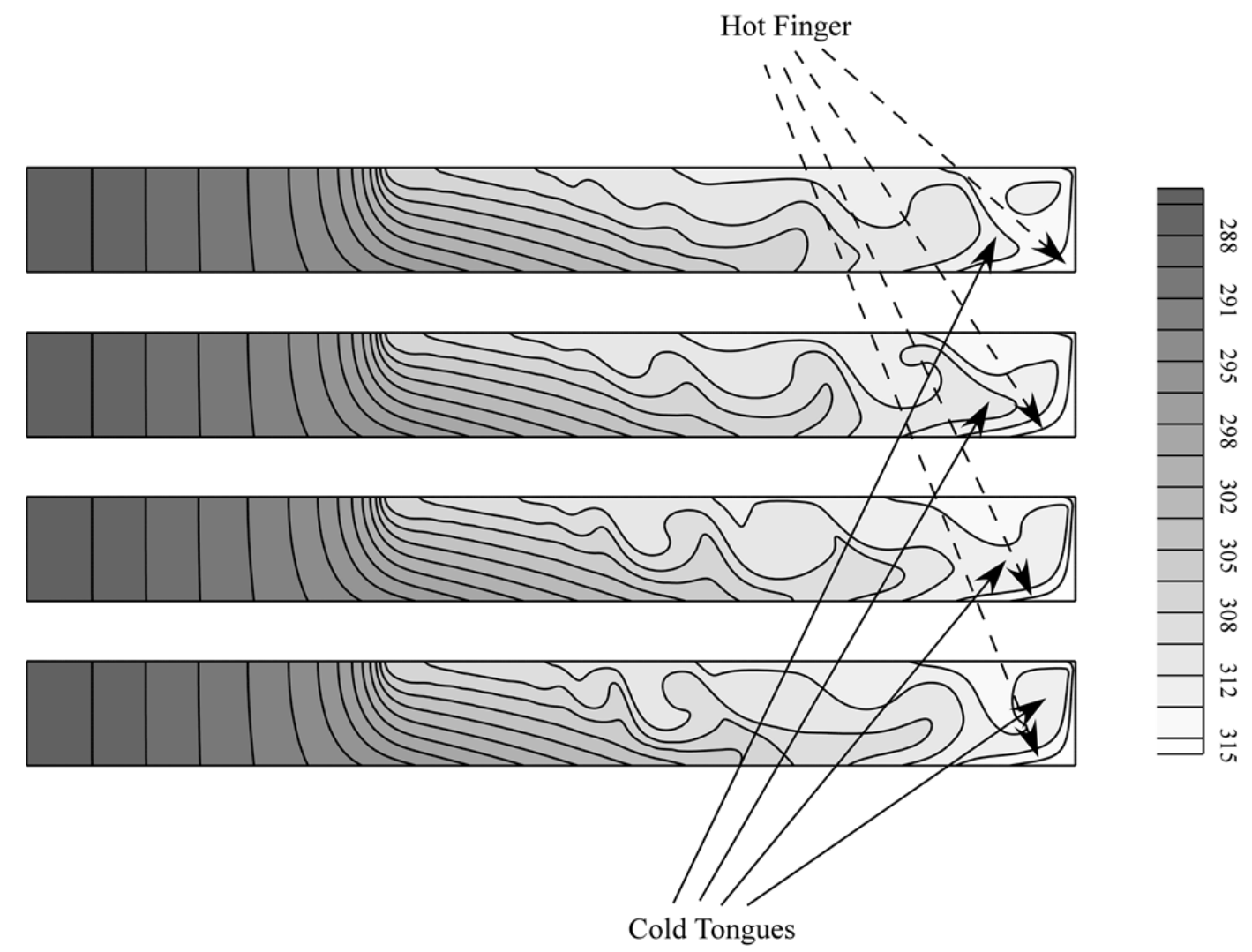

Fig. 24: Snapshots of temperature field for the same conditions considered in Fig. 23.

Figure 24 can be used to provide some additional interesting arguments related to the genesis of this disturbance travelling in the same direction as the surface flow. Interestingly, it can be seen that due to the very inclined configuration of the isotherms in the central region of the layer (maintained by the inclined front of the crystal where temperature is constantly equal to $T_{\text {sol }}$ ), and the additional constraint represented by the bottom conducting boundary (which forces temperature there to maintain a linear profile along the x coordinate), elongated regions of relatively cold fluid are created in the layer where the temperature is at the same time lower than the temperature at the bottom and the temperature at the free liquid-gas surface. Such regions, which protrude in the right warmer part of the layer looking like horizontal "tongues", cause locally a reversal in the sign of the vertical temperature gradient (which can become negative). This effect is particularly striking in proximity to the right sidewall where, owing to the temperature imposed on the bottom and side walls, a hot region (referred to as "hot finger" in Fig. 24) of fluid is established just below a relatively cold tongue coming from the left. The vertical temperature difference between these cold and hot areas tends to become relatively large at the end of the solidification process (in fact, such a difference is not constant in time and increases as the solidification fronts advances towards the hot wall; the more the front advances towards the right, the smaller is the temperature of the cold fingers reaching the hot side). 


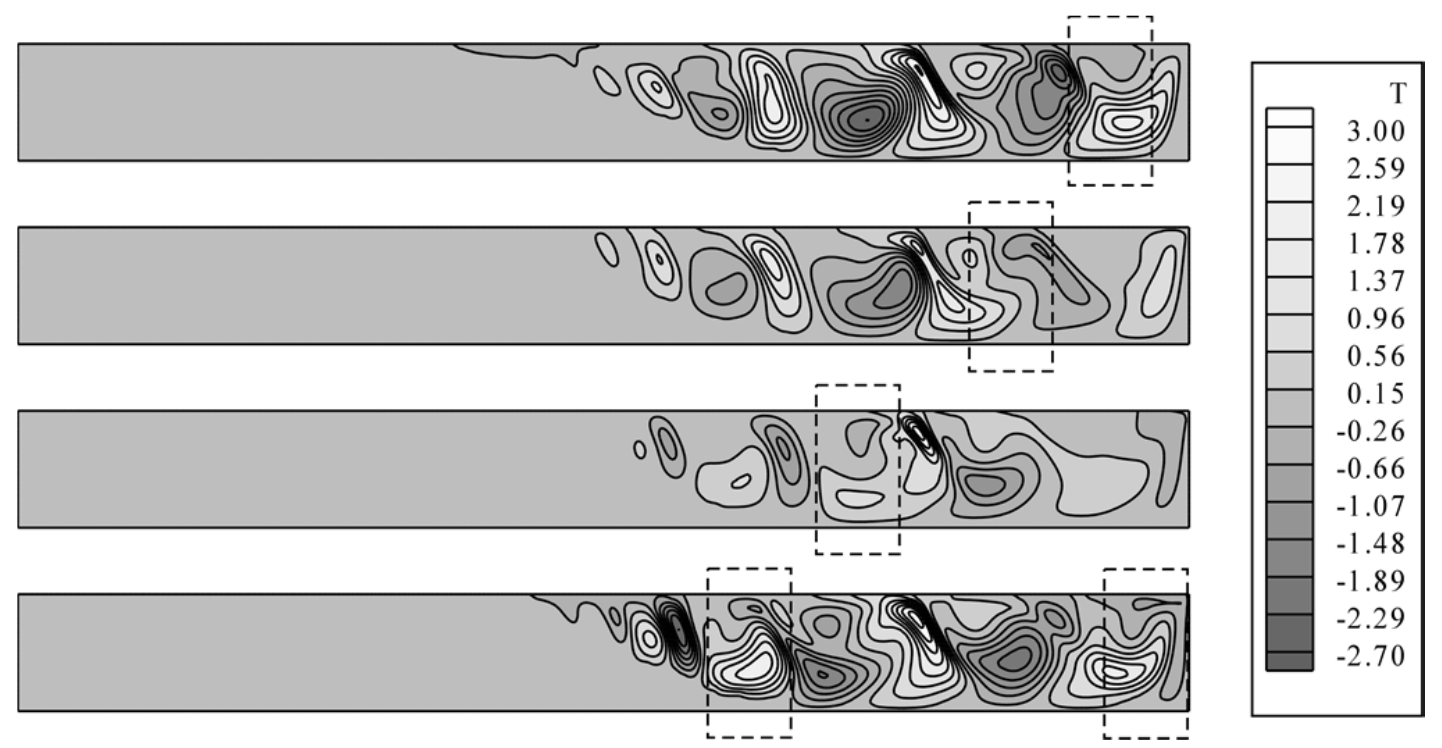

Fig. 25: Snapshots of temperature disturbance for the same conditions considered in Fig. 23.

These effects become even more evident by looking directly at the distribution of temperature disturbance (Fig. 25), where the regions with reversed sign of the vertical gradient of temperature have been highlighted by means of a dashed rectangle.

The existence of such regions can be used to elaborate a reasonable justification for the presence of the disturbance propagating downstream. Indeed, due to the "heating from below" condition that is attained when a portion of relatively hot fluid lies below a cold region, a departure from the classical hydrothermal mechanism typical of Marangoni flow must be expected. Indeed, for such a case a mechanism similar to that driving convection in the classical case of Marangoni-Bénard convection (convection in a layer uniformly heated from below) can be excited.

Such a mechanism can cause locally a convective disturbance on the free liquid-gas interface at those locations where the points located below the interface have a temperature larger than that of surface points at the same horizontal coordinate. Albeit, technically speaking, the Pearson (Marangoni-Bénard) instability is a stationary bifurcation (Pearson [64]), the observed motion of the perturbation (towards the cold side) can be justified taking into account that (as a distinguishing mark with respect to the classical Marangoni-Bénard problem), in the present case the unstructured (initial) base state in the layer is characterized not only by a vertical temperature gradient but also by a symmetry-breaking shear flow (the Marangoni surface stresses driven by the imposed horizontal temperature gradient imply vertical gradients of horizontal velocity). The presence of this shear flow breaks the in-plane isotropy of the usual horizontal layer heated from below, which explains why the perturbations move downstream.

The higher angular frequency of such a perturbation with respect to that of disturbances propagating upstream (of hydrothermal nature) is in line with the conclusions by Shevtsova et al. [65], who identified similar dynamics and underlying cause-and-effect relationships in the case of a Marangoni layer (with no solidification taking place) subjected to a cold gas stream injected from 
the cold side in a direction parallel to the free liquid-gas interface (by lowering surface temperature in proximity to the hot sidewall, the cold tongues in the present case or the cold gas stream in the article by [65], make the thermocapillary layer sensitive to disturbances typical of the MarangoniBénard case).

\section{Conclusions}

While existing research contributions dedicated to purely fluid-dynamic aspects in typical crystalgrowth configurations have been overwhelming, there are some other facets of these problems, which appear to have been inexplicably overlooked. Among these, in primis one should mention the dynamical effects exerted by unsteady solid-melt transition interfaces on the properties of purely surface-tension driven fluid flow and related instabilities (this lack of information being due in large part to the difficulties in preparing laboratory tests that would be at the same time well-controllable and allow exploration of these dynamical effects).

The present paper follows and integrates the line of inquiry started in earlier works where solidification and wave emergence were treated separately. Through a simple numerical model, expressly developed to inquire about the role of the advancing solidification front in changing the properties of the fundamental instability of Marangoni flow, we have investigated how hydrothermal waves can be partially replaced by (or interact with) other fluid-dynamic phenomena. In order to discern the role played by different mechanisms and separate the distinct effects, we have contented ourselves by developing the subject with 2D simulations and have concentrated on a system in which, though the area occupied by the liquid decreases with time, the effective temperature difference along the liquid-gas interface remains constant.

In such conditions the advancing front develops as a strongly inclined boundary 1) protruding into the liquid and 2) causing the number of rolls embedded into the main Marangoni circulation system to increase. Due to the almost horizontal configuration of the front, some smaller-size cells can also be found in the liquid region bounded from below by the crystal. These satellite rolls also contribute to the overall oscillatory behaviour (although their size is much smaller than the other rolls located in the unobstructed part of the cavity). After a certain time depending on the considered substance and cooling rate, an asymptotic regime is attained.

The fixed value of the Marangoni number has proven to be instrumental in unravelling processes that are interwoven or overshadowed in traditional crystal growth processes. Indeed, we could successfully separate the role of the advancing solidification from the other changes in terms of flow spectral content that would be induced by modifications of the (imposed) temperature gradient affecting the liquid phase.

More precisely, we have found that, though the effective horizontal temperature difference is allowed to remain constant, solidification contributes to the chaoticity of the system by exciting new convective modes. Such additional modes are driven by the change in orientation (from horizontal to vertical) of the main temperature gradient established in some regions of the fluid domain. These modes, which share with the classical stationary Pearson (Marangoni-Bénard) 
instability the origin, appear as unsteady disturbances due to the presence of the underlying Marangoni flow, which breaks the isotropy that would be typical of a quiescent layer uniformly heated from below (classical Marangoni-Bénard layer). On the one hand, these disturbances increase the complexity of the frequency spectrum of the asymptotic regime (as made evident by the signals produced by thermocouples placed at fixed locations in the fluid) by causing the emergence of incommensurate frequencies. On the other hand, they can have a remarkable impact on the time-dependent patterning behaviour leading the system to display locally the typical features of a standing wave. Being produced by the overlap of the upstream-travelling HTW and downstream-propagating disturbance of Pearson nature, such a phenomenon is displayed by the system especially in proximity to the heated wall.

Future work shall be devoted to extend such analysis by fully including 3D effects, which may become significant especially for relatively small values of the Prandtl number (namely, $\operatorname{Pr} \leq 10$ ).

\section{Acknowledgement}

We would like to thank Hermes Ferialdi for the kind support provided in executing the frequency spectrum analysis.

\section{References}

[01] Shi W. Y. and Imaishi N., (2006), Hydrothermal waves in differentially heated shallow annular pools of silicone oil, J. Cryst. Growth, 290: 280-291.

[02] Delgado-Buscalioni R., (2001), Convection patterns in end-heated inclined enclosures, Phys. Rev. E 64, 01630317 pages.

[03] Delgado-Buscalioni R., (2002), Effects of thermal boundary conditions and cavity tilt on hydrothermal waves: Suppression of oscillations, Phys. Rev. E, 66: 01630114 pages

[04] Shevtsova V.M., Melnikov D.E., Legros J.C., (2001), Three-dimensional simulations of hydrodynamical instability in liquid bridges: influence of temperature-dependent viscosity, Phys Fluids 13: 2851-2865.

[05] Shevtsova V.M., Melnikov D.E. and Legros J.C., (2003), Multistability of oscillatory thermocapillary convection in a liquid bridge, Phys. Rev. E , 68 (6): 066311 (14 pages).

[06] Schwabe D. and Benz S., (2002), Thermocapillary flow instabilities in an annulus under microgravity - Results of the experiment magia, Adv. Space Res., 29: 629-638.

[07] Schwabe D., Zebib A and Sim B.C., (2003), Oscillatory thermocapillary convection in open cylindrical annuli. Part 1. Experiments under microgravity, J. Fluid Mech., 491: 239-258.

[08] Kaddeche, S., Garandet, J.P., Henry, D., Hadid, H.B., Mojtabi, A., (2015), On the effect of natural convection on solute segregation in the horizontal Bridgman configuration: 
Convergence of a theoretical model with numerical and experimental data, J. Cryst. Growth, 409: 89-94.

[09] Pimputkar S.M. and Ostrach S., (1981), Convective effects in crystals grown from melt, J. Cryst. Growth, 55(3): 614-646

[10] Müller G., (1993), Convective instabilities in melt growth configurations, J. Cryst. Growth, $128(1-4): 26-36$

[11] Ohta H., Nomura K., Hiramatsu H., Ueda K., Kamiya T., Hirano M., Hosono H., (2003), Frontier of transparent oxide semiconductors, Solid-State Electronics, 47: 2261-2267.

[12] Stadler A., (2012), Transparent Conducting Oxides-An Up-To-Date Overview, Materials, 5: 661-683.

[13] Lappa M., (2005), Thermal convection and related instabilities in models of crystal growth from the melt on earth and in microgravity: Past history and current status, Cryst. Res. Technol., 40(6): 531-549.

[14] Lappa M., Thermal Convection: Patterns, Evolution and Stability (John Wiley \& Sons, Chichester, England, 2009).

[15] Coriell S.R., McFadden G.B., Boisvert R.F., Glicksman M.E., Fang Q.T., (1984), Coupled convective instabilities at crystal-melt interfaces, J. Cryst. Growth, 66(3): 514-524.

[16] Lan, C. W., Chen, M. K. and Liang, M. C. (1998) Bifurcation and stability analyses of horizontal Bridgman crystal growth of a low Prandtl number material, J. Cryst. Growth, 187 (2): 303-313.

[17] Bertrand O., Binet B., Combeau H., Couturier S., Delannoy Y., Gobin D., Lacroix M., Le Quéré P., Médale M., Mencinger J., Sadat H., Vieira G., (1999), Melting driven by natural convection A comparison exercise: first results, International Journal of Thermal Sciences, 38(1): 5-26.

[18] Tönhardt R. and Amberg G., (2000), Simulation of natural convection effects on succinonitrile crystals, Phys. Rev. E 62, 828.

[19] El Ganaoui, M., Lamazouade, A., Bontoux, P. and Morvan, D. (2002) Computational solution for fluid flow under solid/liquid phase change conditions, Comput. Fluids, 31 (4-7), $539-556$.

[20] Semma, E., El Ganaoui, M., Cheddadi, A. and Bontoux, P. (2003) Etude numérique des instabilités de la phase fluide et de l'interface de solidification en croissance dirigée horizontale, C. R. Acad. Sci., Paris, 331 (9): 631-639.

[21] Stickland M.T., Scanlon T.J., MacKenzie J., (2007), An experimental investigation of natural convection with solidification in a differentially heated cavity, International Journal of Heat and Mass Transfer 50: 36-44. 
[22] Wang S., Faghri A., Bergman T. L., (2010), A comprehensive numerical model for melting with natural convection, International Journal of Heat and Mass Transfer, 53(9-10): 19862000.

[23] Shyy W. and Chen M.H.. (1991), Interaction of thermocapillary and natural convection flows during solidification: normal and reduced gravity conditions, J. Crystal Growth, 108: 247-261.

[24] Lan C.W. and Kou S., (1991), Heat transfer, fluid flow and interface shapes in floating-zone crystal growth, J. Cryst. Growth, 108 (1-2): 351-366

[25] Cerimele M. M., Mansutti D., Pistella F., (2003), Marangoni effects in a horizontal solidification process in microgravity, ICCMSE '03 Proceedings of the international conference on Computational methods in sciences and engineering, Pages 110-113, World Scientific Publishing Co., Inc. River Edge, NJ, USA (2003).

[26] Amberg G. and Shiomi J., (2005), Thermocapillary Flow and Phase Change in Some Widespread Materials Processes, Fluid Dynamics \& Materials Processing, 1(1): 81-95.

[27] Tsukada T., Kobayashi M., Jing C. J., and Imaishi N., (2005), Numerical Simulation of CZ Crystal Growth of Oxide, Fluid Dynamics \& Materials Processing, 1(1): 45-62

[28] Matsunaga K. and Kawamura H., (2006), Influence of Thermocapillary Convection on Solid-liquid Interface, Fluid Dynamics Materials Processing, 2(1): 59-64.

[29] Hong Y., Jin W.Q., Pan X.H., Shinichi Y., (2006), Experimental study on marangoni convection and solidification in $\mathrm{BaB} 2 \mathrm{O} 4$ melt, International Journal of Heat and Mass Transfer, 49(21-22): 4254-4257.

[30] Lan C.W., Chian C.H., (2001), Three-dimensional simulation of Marangoni flow and interfaces in floating-zone silicon crystal growth, J. Cryst. Growth, 230: 172-180.

[31] Lappa M. and Savino R., (2002), 3D analysis of crystal/melt interface shape and Marangoni flow instability in solidifying liquid bridges, Journal of Computational Physics, 180 (2): 751 774

[32] Lappa M., (2016), On the onset of multi-wave patterns in laterally heated floating zones for slightly supercritical conditions, Physics of Fluids, 28(12): 124105 (22 pages)

[33] Smith M.K. and Davis S.H. (1983), Instabilities of dynamic thermocapillary liquid layers. Part 1: convective instabilities, J. Fluid Mech 132: 119-144.

[34] Peltier L. and Biringen S., (1993), Time-dependent thermocapillary convection in a rectangular cavity: numerical results for a moderate Prandtl number fluid, J. Fluid Mech., 257: 339-357.

[35] $\mathrm{Xu} \mathrm{J.} \mathrm{and} \mathrm{Zebib} \mathrm{A.,} \mathrm{(1998),} \mathrm{Oscillatory} \mathrm{two-} \mathrm{and} \mathrm{three-dimensional} \mathrm{thermocapillary}$ convection, J. Fluid Mech., 364: 187-209. 
[36] Shevtsova V.M., Nepomnyashchy A.A. and Legros J.C., (2003), Thermocapillary-buoyancy convection in a shallow cavity heated from the side, Phys. Rev. E 67, 066308 (14 pages).

[37] Tang Z.M. and Hu W.R., (2005), Hydrothermal Wave in a Shallow Liquid Layer, Microgravity Sci. Tech., 16(1): 253-258.

[38] Lappa M., (2016), Patterning behaviour of gravitationally modulated supercritical Marangoni flow in liquid layers, Phys. Rev. E, 93(5), 053107, (13 pages).

[39] Bucchignani E. and Mansutti D., (2004), Horizontal thermocapillary convection of succinonitrile: Steady state, instabilities, and transition to chaos, Phys. Rev. E 69, 056319.

[40] Smith M.K, (1986), Instability mechanism in dynamic thermocapillary liquid layers, Phys. Fluids, 29 (10): 3182-3186.

[41] Priede J. and Gerbeth G., (1997), Influence of thermal boundary conditions on the stability of thermocapillary-driven convection at low Prandtl numbers, Phys. Fluids, 9: 1621-1634.

[42] Lappa M., (2017), Hydrothermal waves in two-dimensional liquid layers with sudden changes in the available cross-section, Int. J. Num. Meth. Heat Fluid Flow, 27 (11): 26292649.

[43] Voller V. R., Prakash C., (1987), A fixed grid numerical modelling methodology for convection-diffusion mushy region phase-change problems, Int. J. Heat Mass Transfer, 30 (8): 1709-1719.

[44] Bennon W.D., Incropera F.P., (1987), A continuum model for momentum, heat and species transport in binary solid-liquid phase change systems-I. Model formulation, Int.J.Heat Mass Transfer, 30 (10): 2161-2170.

[45] Bennon W.D., Incropera F.P. , (1987), A continuum model for momentum, heat and species transport in binary solid-liquid phase change systems-II. Application to solidification in a rectangular cavity, Int. J.Heat Mass Transfer, 30 (10): 2171-2187.

[46] Brent A.D., Voller V.R., Reid J., (1988), Enthalpy-porosity technique for modelling convection-diffusion phase change: application to the melting of a pure metal, Num. Heat Transf., 13: 297-318.

[47] Beckermann C., Diepers H.-J., Steinbach I., Karma A. and Tong X., (1999), Modeling melt convection in phase-field simulations of solidification, J. Comput. Phys., 154 (2): 468-496.

[48] Knoll D., Kothe D., Lally B., (1999), A New Nonlinear Solution Method for Phase Change Problems, Num. Heat Transfer Part B, 35 (4): 439-459.

[49] Rao M. M., Shyy W., (1997), Moving boundary computation of the float-zone process, Int. J. Numer. Meth. Eng., 40 (7): 1231-1261

[50] Lan C.W., Liang M.C., (1999), Multigrid methods for incompressible heat flow problems with an unknown interface, J. Comput. Phys., 152 (1): 55-77. 
[51] Lan C.W., Liu C.C., et al., (2002), An adaptive finite volume method for incompressible heat flow problems in solidification, J. Comput. Phys., 178 (2): 464-471.

[52] Udaykumar H. S., Mittal R., Shyy W., (1999), Computation of solid-liquid phase fronts in the sharp interface limit on fixed grids, J. Comput. Phys., 153 (2): 535-574.

[53] Kim Y.T., Goldenfeld N., Dantzig J., (2000), Computation of dendritic microstructures using a level set method, Phys. Rev. E, 62: 2471-2474.

[54] Sekerka R.F., (2004), Morphology: from sharp interface to phase field models, J. Cryst. Growth, 264(4): 530-540.

[55] Faiez R., and Rezaei Y., (2017), A Single-domain Approach to Simulate the Effect of Convective Flow on the Mushy-zone Structure in Czochralski Growth of Gadolinium Gallium Garnet Crystal, Universal Journal of Materials Science 5(3): 73-81, DOI: 10.13189/ujms.2017.050303

[56] Lee H. and Pearlstein A.J., (2000), Simulation of vertical Bridgman growth of benzene, a material with anisotropic solid-phase thermal conductivity, J Cryst. Growth, 209, 934-952.

[57] Wang L., Rhee H., Felicelli S. D., Sabau A.S., Berry J.T., (2010), Interdependence between cooling rate, microstructure and porosity in MG alloy AE42, in Magnesium Technology, edited by Eric A. Nyberg, Sean R. Agnew, Neale R. Neelameggham, Mihriban O. Pekguleryuz, John Wiley \& Sons (2010).

[58] Michalik R., Woźnica H., Tomaszewska A., (2013), Effect of cooling rate on the porosity of the ZnAl22Cu3 alloy, Solid State Phenomena, 197, 221-225, doi:10.4028/www.scientific.net/SSP.197.221

[59] Brůna M., Bolibruchová D., Pastirčák R., (2017), Numerical simulation of porosity for Al based alloys, Procedia Engineering 177, 488-495.

[60] Kasperski G., Labrosse G., (2000), On the numerical treatment of viscous singularities in wall-confined thermocapillary convection, Phys. Fluids, 12(11): 2695-2697.

[61] Melnikov D., Pushkin D., and Shevtsova V., (2011), Accumulation of particles in timedependent thermocapillary flow in a liquid bridge. Modeling of experiments, Eur. Phys. J. Special Topics, 192: 29-39

[62] Nissen D. A., and Meeker D. E., (1983), Nitrate Nitrite Chemistry in NaNO3-KNO3 melts, Inorganic Chemistry, 22(5): 716-721.

[63] Chen Z., Chen C.L., Hao L.M., (2008), Numerical simulation of succinonitrile dendritic growth in a forced flow, Acta Metall. Sin., 21 (6): 444-450

[64] Pearson, J.R.A., (1958), On convection cells induced by surface tension, J.Fluid Mech., 4: 489-500. 
[65] Shevtsova V., Gaponenko Y., Kuhlmann H.C., Lappa M., Lukasser M., Matsumoto S., Mialdun A., Montanero J.M., Nishino K., and Ueno I., (2014), The JEREMI-Project on Thermocapillary Convection in Liquid Bridges. Part B: Impact of Co-axial Gas Flow, Fluid Dynamics \& Materials Processing, 10(2), 197-240. 